\title{
Measurements of the strong coupling constant and the QCD colour factors using four-jet observables from hadronic $Z$ decays
}

\author{
Journal Article \\ Author(s): \\ The ALEPH Collaboration; Heister, Arno; Dissertori, Günther; et al. \\ Publication date: \\ 2003-03 \\ Permanent link: \\ https://doi.org/10.3929/ethz-b-000056466
}

Rights / license:

Creative Commons Attribution 4.0 International

Originally published in:

The European Physical Journal C 27(1), https://doi.org/10.1140/epjc/s2002-01114-2 


\section{Measurements of the strong coupling constant and the QCD colour factors using four-jet observables from hadronic $Z$ decays}

The ALEPH Collaboration

\section{A. Heister, S. Schael}

Physikalisches Institut das RWTH-Aachen, 52056 Aachen, Germany

R. Barate, I. De Bonis, D. Decamp, C. Goy, J.-P. Lees, E. Merle, M.-N. Minard, B. Pietrzyk Laboratoire de Physique des Particules (LAPP), IN ${ }^{2} \mathrm{P}^{3}$-CNRS, 74019 Annecy-le-Vieux Cedex, France

G. Boix ${ }^{24}$, S. Bravo, M.P. Casado, M. Chmeissani, J.M. Crespo, E. Fernandez, M. Fernandez-Bosman, Ll. Garrido ${ }^{15}$, E. Graugés, M. Martinez, G. Merino, R. Miquel ${ }^{27}$, Ll.M. Mir²7, A. Pacheco, H. Ruiz Institut de Física d'Altes Energies, Universitat Autònoma de Barcelona, 08193 Bellaterra (Barcelona), Spain ${ }^{7}$

A. Colaleo, D. Creanza, M. de Palma, G. Iaselli, G. Maggi, M. Maggi, S. Nuzzo, A. Ranieri, G. Raso ${ }^{23}$, F. Ruggieri, G. Selvaggi, L. Silvestris, P. Tempesta, A. Tricomi ${ }^{3}$, G. Zito

Dipartimento di Fisica, INFN Sezione di Bari, 70126 Bari, Italy

X. Huang, J. Lin, Q. Ouyang, T. Wang, Y. Xie, R. Xu, S. Xue, J. Zhang, L. Zhang, W. Zhao Institute of High Energy Physics, Academia Sinica, Beijing, The People's Republic of China ${ }^{8}$

D. Abbaneo, P. Azzurri, O. Buchmüller ${ }^{25}$, M. Cattaneo, F. Cerutti, B. Clerbaux, H. Drevermann, R.W. Forty, M. Frank, F. Gianotti, T.C. Greening ${ }^{29}$, J.B. Hansen, J. Harvey, D.E. Hutchcroft, P. Janot, B. Jost, M. Kado ${ }^{31}$, P. Mato, A. Moutoussi, F. Ranjard, L. Rolandi, D. Schlatter, O. Schneider ${ }^{2}$, G. Sguazzoni, W. Tejessy, F. Teubert, A. Valassi, I. Videau, J. Ward

European Laboratory for Particle Physics (CERN), 1211 Geneva 23, Switzerland

F. Badaud, A. Falvard ${ }^{22}$, P. Gay, P. Henrard, J. Jousset, B. Michel, S. Monteil, J-C. Montret, D. Pallin, P. Perret Laboratoire de Physique Corpusculaire, Université Blaise Pascal, IN ${ }^{2} \mathrm{P}^{3}$-CNRS, Clermont-Ferrand, 63177 Aubière, France

J.D. Hansen, J.R. Hansen, P.H. Hansen, B.S. Nilsson

Niels Bohr Institute, 2100 Copenhagen, Denmark ${ }^{9}$

A. Kyriakis, C. Markou, E. Simopoulou, A. Vayaki, K. Zachariadou

Nuclear Research Center Demokritos (NRCD), 15310 Attiki, Greece

A. Blondel ${ }^{12}$, G. Bonneaud, J.-C. Brient, A. Rougé, M. Rumpf, M. Swynghedauw, M. Verderi,

H. Videau

Laboratoire de Physique Nucléaire et des Hautes Energies, Ecole Polytechnique, IN ${ }^{2} \mathrm{P}^{3}$-CNRS, 91128 Palaiseau Cedex, France

V. Ciulli, E. Focardi, G. Parrini

Dipartimento di Fisica, Università di Firenze, INFN Sezione di Firenze, 50125 Firenze, Italy

A. Antonelli, M. Antonelli, G. Bencivenni, F. Bossi, P. Campana, G. Capon, V. Chiarella, P. Laurelli, G. Mannocchi ${ }^{5}$, F. Murtas, G.P. Murtas, L. Passalacqua, M. Pepe-Altarellii ${ }^{4}$

Laboratori Nazionali dell'INFN (LNF-INFN), 00044 Frascati, Italy

A. Halley, J.G. Lynch, P. Negus, V. O'Shea, C. Raine ${ }^{32}$, A.S. Thompson

Department of Physics and Astronomy, University of Glasgow, Glasgow G12 8QQ, UK ${ }^{10}$

S. Wasserbaech

Department of Physics, Haverford College, Haverford, PA 19041-1392, USA

R. Cavanaugh, S. Dhamotharan, C. Geweniger, P. Hanke, V. Hepp, E.E. Kluge, A. Putzer, H. Stenzel, K. Tittel, M. Wunsch ${ }^{19}$

Kirchhoff-Institut für Physik, Universität Heidelberg, 69120 Heidelberg, Germany ${ }^{16}$

R. Beuselinck, D.M. Binnie, W. Cameron, P.J. Dornan, M. Girone ${ }^{1}$, N. Marinelli, J.K. Sedgbeer, J.C. Thompson ${ }^{14}$ Department of Physics, Imperial College, London SW7 2BZ, UK ${ }^{10}$

V.M. Ghete, P. Girtler, E. Kneringer, D. Kuhn, G. Rudolph

Institut für Experimentalphysik, Universität Innsbruck, 6020 Innsbruck, Austria ${ }^{18}$ 
E. Bouhova-Thacker, C.K. Bowdery, A.J. Finch, F. Foster, G. Hughes, R.W.L. Jones, M.R. Pearson, N.A. Robertson Department of Physics, University of Lancaster, Lancaster LA1 4YB, UK ${ }^{10}$

K. Jakobs, K. Kleinknecht, G. Quast ${ }^{6}$, B. Renk, H.-G. Sander, H. Wachsmuth, C. Zeitnitz Institut für Physik, Universität Mainz, 55099 Mainz, Germany ${ }^{16}$

A. Bonissent, P. Coyle, O. Leroy, P. Payre, D. Rousseau, M. Talby

Centre de Physique des Particules, Université de la Méditerranée, IN² $\mathrm{P}^{3}$-CNRS, 13288 Marseille, France

F. Ragusa

Dipartimento di Fisica, Università di Milano e INFN Sezione di Milano, 20133 Milano, Italy

A. David, H. Dietl, G. Ganis ${ }^{26}$, K. Hüttmann, G. Lütjens, W. Männer, H.-G. Moser, R. Settles, W. Wiedenmann, G. Wolf

Max-Planck-Institut für Physik, Werner-Heisenberg-Institut, 80805 München, Germany ${ }^{16}$

J. Boucrot, O. Callot, M. Davier, L. Duflot, J.-F. Grivaz, Ph. Heusse, A. Jacholkowska ${ }^{30}$, J. Lefrançois, J.-J. Veillet, C. Yuan

Laboratoire de l'Accélérateur Linéaire, Université de Paris-Sud, IN² $\mathrm{P}^{3}$-CNRS, 91898 Orsay Cedex, France

G. Bagliesi, T. Boccali, L. Foà, A. Giammanco, A. Giassi, F. Ligabue, A. Messineo, F. Palla, G. Sanguinetti, A. Sciabà, P. Spagnolo, R. Tenchini ${ }^{1}$, A. Venturi ${ }^{1}$, P.G. Verdini, Z. Xie

Dipartimento di Fisica dell'Università, INFN Sezione di Pisa, e Scuola Normale Superiore, 56010 Pisa, Italy

G.A. Blair, G. Cowan, M.G. Green, T. Medcalf, A. Misiejuk, J.A. Strong, P. Teixeira-Dias,

Department of Physics, Royal Holloway \& Bedford New College, University of London, Egham, Surrey TW20 OEX, UK ${ }^{10}$

R.W. Clifft, T.R. Edgecock, P.R. Norton, I.R. Tomalin

Particle Physics Dept., Rutherford Appleton Laboratory, Chilton, Didcot, Oxon OX11 OQX, UK ${ }^{10}$

B. Bloch-Devaux, P. Colas, S. Emery, W. Kozanecki, E. Lançon, M.-C. Lemaire, E. Locci, P. Perez, J. Rander, J.-F. Renardy, A. Roussarie, J.-P. Schuller, J. Schwindling, A. Trabelsi ${ }^{21}$, B. Vallage

CEA, DAPNIA/Service de Physique des Particules, CE-Saclay, 91191 Gif-sur-Yvette Cedex, France ${ }^{17}$

N. Konstantinidis, A.M. Litke, G. Taylor

Institute for Particle Physics, University of California at Santa Cruz, Santa Cruz, CA 95064, USA ${ }^{13}$

C.N. Booth, S. Cartwright, F. Combley ${ }^{32}$, M. Lehto, L.F. Thompson

Department of Physics, University of Sheffield, Sheffield S3 7RH, UK ${ }^{10}$

K. Affholderbach ${ }^{28}$, A. Böhrer, S. Brandt, C. Grupen, A. Ngac, G. Prange, U. Sieler

Fachbereich Physik, Universität Siegen, 57068 Siegen, Germany ${ }^{16}$

G. Giannini

Dipartimento di Fisica, Università di Trieste e INFN Sezione di Trieste, 34127 Trieste, Italy

J. Rothberg

Experimental Elementary Particle Physics, University of Washington, Seattle, WA 98195, USA

S.R. Armstrong, K. Berkelman, K. Cranmer, D.P.S. Ferguson, Y. Gao ${ }^{20}$, S. González, O.J. Hayes, H. Hu, S. Jin, J. Kile, P.A. McNamara III, J. Nielsen, Y.B. Pan, J.H. von Wimmersperg-Toeller, W. Wiedenmann, J. Wu, Sau Lan $\mathrm{Wu}, \mathrm{X} . \mathrm{Wu}, \mathrm{G}$. Zobernig

Department of Physics, University of Wisconsin, Madison, WI 53706, USA ${ }^{11}$

G. Dissertori

Institute for Particle Physics, ETH Hönggerberg, 8093 Zürich, Switzerland

Received: 14 May 2002 /

Published online: 15 January 2003 - (c) Springer-Verlag / Società Italiana di Fisica 2003

Abstract. Data from $\mathrm{e}^{+} \mathrm{e}^{-}$annihilation into hadrons, taken with the ALEPH detector at the $\mathrm{Z}$ resonance, are analyzed. The four-jet rate is studied as a function of the resolution parameter and compared to next-toleading order calculations combined with resummation of large logarithms. Angular correlations in four-jet events are measured and compared to next-to-leading order QCD predictions. With these observables two different measurements are performed. In a first analysis the strong coupling constant is measured from the four-jet rate yielding

$$
\alpha_{s}\left(M_{\mathrm{Z}}\right)=0.1170 \pm 0.0001(\text { stat }) \pm 0.0013(\text { sys }) .
$$


In a second measurement the strong coupling constant and the QCD colour factors are determined simultaneously from a fit to the four-jet rate and the four-jet angular correlations, giving

$$
\begin{gathered}
\alpha_{s}\left(M_{\mathrm{Z}}\right)=0.119 \pm 0.006(\text { stat }) \pm 0.026(\mathrm{sys}) \\
C_{\mathrm{A}}=2.93 \pm 0.14(\text { stat }) \pm 0.58(\mathrm{sys}) \\
C_{\mathrm{F}}=1.35 \pm 0.07(\text { stat }) \pm 0.26(\text { sys })
\end{gathered}
$$

in good agreement with the expectation from QCD. 


\footnotetext{
1 Also at CERN, 1211 Geneva 23, Switzerland

2 Now at Université de Lausanne, 1015 Lausanne, Switzerland

3 Also at Dipartimento di Fisica di Catania and INFN Sezione di Catania, 95129 Catania, Italy

4 Now at CERN, 1211 Geneva 23, Switzerland

5 Also Istituto di Cosmo-Geofisica del C.N.R., Torino, Italy

6 Now at Institut für Experimentelle Kernphysik, Universität Karlsruhe, 76128 Karlsruhe, Germany

7 Supported by CICYT, Spain

8 Supported by the National Science Foundation of China

9 Supported by the Danish Natural Science Research Council

10 Supported by the UK Particle Physics and Astronomy Research Council

11 Supported by the US Department of Energy, grant DEFG0295-ER40896

12 Now at Departement de Physique Corpusculaire, Université de Genève, 1211 Genève 4, Switzerland

13 Supported by the US Department of Energy, grant DEFG03-92ER40689

14 Supported by the Leverhulme Trust
}

15 Permanent address: Universitat de Barcelona, 08208 Barcelona, Spain

16 Supported by the Bundesministerium für Bildung, Wissenschaft, Forschung und Technologie, Germany

17 Supported by the Direction des Sciences de la Matière, C.E.A

18 Supported by the Austrian Ministry for Science and Transport

19 Now at SAP AG, 69185 Walldorf, Germany

20 Also at Department of Physics, Tsinghua University, Beijing, The People's Republic of China

${ }^{21}$ Now at Département de Physique, Faculté des Sciences de Tunis, 1060 Le Belvédère, Tunisia

22 Now at Groupe d'Astroparticules de Montpellier, Université de Montpellier II, 34095, Montpellier, France

23 Also at Dipartimento di Fisica e Tecnologie Relative, Università di Palermo, Palermo, Italy

24 Now at McKinsey and Compagny, Avenue Louis Casal 18, 1203 Geneva, Switzerland

25 Now at SLAC, Stanford, CA 94309, USA

26 Now at INFN Sezione di Roma II, Dipartimento di Fisica, Universitá di Roma Tor Vergata, 00133 Roma, Italy

27 Now at LBNL, Berkeley, CA 94720, USA

28 Now at Skyguide, Swissair Navigation Services, Geneva, Switzerland

29 Now at Honeywell, Phoenix AZ, USA

30 Also at Groupe d'Astroparticules de Montpellier, Université de Montpellier II, 34095, Montpellier, France

31 Now at Fermilab, PO Box 500, MS 352, Batavia, IL 60510, USA

32 Deceased

\section{Introduction}

Electron-positron annihilation into hadrons at high energy is a well suited process to test Quantum Chromodynamics (QCD) since the initial state is well known and longdistance effects are typically small. Many QCD studies have been carried out at LEP, in particular precise measurements of the strong coupling constant $\alpha_{s}\left(M_{\mathrm{Z}}\right)[1]$ and tests of the structure of the underlying gauge group [2,3], which is $\mathrm{SU}(3)$ in the case of QCD.

Four-jet events are particularly interesting, since QCD shows its full gauge structure only from order $\alpha_{s}^{2}$ on. The gluon self-coupling, a consequence of the non-abelian nature of QCD, leads to an $\mathrm{e}^{+} \mathrm{e}^{-} \rightarrow \mathrm{q} \overline{\mathrm{q} g g}$ cross section that dominates over the $\mathrm{e}^{+} \mathrm{e}^{-} \rightarrow \mathrm{q} \overline{\mathrm{q}} \mathrm{q}^{\prime} \overline{\mathrm{q}}^{\prime}$ process. Thus the measurement of multi-jet rates could be expected to distinguish between abelian and non-abelian hypotheses. However, a much more powerful method for testing these hypotheses makes use of the different correlations among the final-state particles induced by the various contributions to the cross section. These correlations have their origin in the different angular momentum properties of the final state.

Here both the four-jet rate and four-jet angular correlations are studied. The four-jet rate is very sensitive to the strong coupling constant and, as the resummation of large logarithms exists, its dependence on the renormalization scale is heavily reduced. For the angular observables the sensitivity to the QCD colour factors is exploited. Their sensitivity to the strong coupling constant is reduced by normalizing the distributions to unit area. This normalization is performed in order to suppress the strong scale dependence given by the lack of the resummation of large logarithms for this set of observables.

Two different measurements are performed. In a first measurement the strong coupling constant is obtained from the four-jet rate. Then, a combined measurement of the strong coupling constant and the colour factors using next-to-leading order (NLO) calculations [4-11] is obtained, by fitting the resummed NLO predictions for the four-jet rate and the normalized NLO predictions for the four-jet angular correlations to ALEPH data.

In the following section the theoretical framework is summarized, after which the ALEPH detector and the data analysis are described. After introducing the method used for the measurements, details are shown of the determination of the strong coupling constant from the four-jet rate, and of the combined fit of the strong coupling constant and the QCD colour factors from four-jet observables. Finally the conclusions are given. 


\section{Observables and theoretical predictions}

The NLO differential cross section for a four-jet observable, $\mathrm{O}_{4}$, can be written as,

$$
\begin{aligned}
& \frac{1}{\sigma_{0}} \frac{\mathrm{d} \sigma}{\mathrm{d} O_{4}}\left(O_{4}\right)=\eta(\mu)^{2} B_{4}\left(O_{4}\right) \\
& \quad+\eta(\mu)^{3}\left[B_{4}\left(O_{4}\right) \beta_{0} \ln x_{\mu}^{2}+C_{4}\left(O_{4}\right)\right]+\mathcal{O}\left(\eta^{4}\right)
\end{aligned}
$$

with

$$
\eta(\mu)=\left(\frac{\alpha_{s}(\mu) C_{F}}{2 \pi}\right)
$$

where $\sigma_{0}$ is the Born cross section for $\mathrm{e}^{+} \mathrm{e}^{-}$annihilation into hadrons, $\mu$ is the renormalization scale, $x_{\mu}$ the ratio of $\mu$ with respect to the $\mathrm{Z}$ boson mass, and $B_{4}$ and $C_{4}$ are the Born and NLO scale-independent functions, respectively. They are obtained from the integration of the fully differential massless matrix elements for $\mathrm{e}^{+} \mathrm{e}^{-}$annihilation into four-parton final states. In this paper the integration has been carried out with the Monte Carlo (MC) program DEBRECEN 2.0 [12] by generating more than 100 million events.

For the running coupling the two-loop expression

$$
\eta(\mu)=\frac{\eta\left(M_{\mathrm{Z}}\right)}{w(\mu)}\left(1-\frac{\beta_{1}}{\beta_{0}} \eta\left(M_{\mathrm{Z}}\right) \frac{\ln w(\mu)}{w(\mu)}\right)
$$

is used, with

$$
\begin{aligned}
w(\mu) & =1-\beta_{0} \eta\left(M_{\mathrm{Z}}\right) \ln \left(\frac{M_{\mathrm{Z}}}{\mu}\right), \\
\beta_{0} & =\frac{11}{3} x-\frac{4}{3} y N_{f}, \\
\beta_{1} & =\frac{17}{3} x^{2}-2 y N_{f}-\frac{10}{3} x y N_{f} .
\end{aligned}
$$

The variables $x=\frac{C_{A}}{C_{F}}$ and $y=\frac{T_{R}}{C_{F}}$ are the QCD colour factor ratios, and $N_{f}=5$ is the number of active flavours. Using the expected values from $\mathrm{SU}(3)$ for the colour factors, $C_{A}=3$ and $C_{F}=4 / 3$, together with the normalization $T_{R}=1 / 2$, the theoretical prediction for the ratios is $x=2.25$ and $y=0.375$.

The measurement of the colour factors using four-jet observables is possible thanks to the linear and quadratic dependence of the $B$ and $C$ functions,

$$
\begin{aligned}
B_{4}= & B_{0}+B_{x} x+B_{y} y, \\
C_{4}= & C_{0}+C_{x} x+C_{y} y+C_{z} z \\
& +C_{x x} x^{2}+C_{x y} x y+C_{y y} y^{2},
\end{aligned}
$$

where $z=\frac{C_{3}}{N_{c} C_{F}^{3}} . C_{3}$ is the square of a cubic Casimir operator and $N_{c}$ is the number of colours. The $C_{z}$ functions have been found to be very small, of the order of one permille compared to $C_{4}$, and therefore are not taken into account in the present study.

Expression (1) is used to predict the four-jet rate $\left(R_{4}\right)$ at NLO as a function of the clustering resolution parameter $y_{\text {cut }}$. In order to obtain the proper normalization the relation $\sigma_{\text {tot }}=\sigma_{0}\left(1+\frac{3}{2} \eta\right)$ is used. Jets are defined by the Durham clustering algorithm with the E-recombination scheme (Durham-E clustering) [13]. Four-jet fractions decrease very rapidly when increasing the resolution parameter, so most of the data is found at small $y_{\text {cut }}$. However, the fixed-order perturbative prediction is not reliable for small values of $y_{\text {cut }}$, due to terms $\alpha_{s}^{n} \ln ^{m} y_{\text {cut }}$ that enhance the higher order corrections. The all-order resummation of the leading and next-to-leading logarithmic (NLL) contributions has to be performed. This resummation is possible with the Durham clustering algorithm using the coherent branching formalism. The expression for the four-jet rate in the NLL approximation is given in [11]. As the Durham four-jet rate can be resummed but does not satisfy a simple exponentiation, the only viable matching schemes are the $\mathrm{R}$ matching or the modified $\mathrm{R}$ matching $([14,15])$. Here the $\mathrm{R}$ matching is employed following again [11].

The following four-jet angular distributions are calculated for selected four-jet events, again with the Durham-E clustering algorithm, at $y_{\text {cut }}=0.008$ :

- the Bengtsson-Zerwas angle [16]; $\left|\cos \left(\chi_{\mathrm{BZ}}\right)\right|=\left|\cos \left(\angle\left[\left(\vec{p}_{1} \times \vec{p}_{2}\right),\left(\vec{p}_{3} \times \vec{p}_{4}\right)\right]\right)\right|$

- the Körner-Schierholtz-Willrodt angle [17];

$$
\begin{aligned}
& \quad \cos \left(\Phi_{\mathrm{KSW}}\right)=\cos \left(\frac { 1 } { 2 } \left(\angle\left[\left(\vec{p}_{1} \times \vec{p}_{4}\right),\left(\vec{p}_{2} \times \vec{p}_{3}\right)\right]\right.\right. \\
& \left.\left.\quad+\angle\left[\left(\vec{p}_{1} \times \vec{p}_{3}\right),\left(\vec{p}_{2} \times \vec{p}_{4}\right)\right]\right)\right) \\
& - \text { the modified Nachtmann-Reiter angle }[18] ; \\
& \quad\left|\cos \left(\Theta_{\mathrm{NR}}\right)\right|=\left|\cos \left(\angle\left[\left(\vec{p}_{1}-\vec{p}_{2}\right),\left(\vec{p}_{3}-\vec{p}_{4}\right)\right]\right)\right| \\
& - \text { the angle between the two lowest energetic jets }[19] ; \\
& \quad \cos \left(\alpha_{34}\right)=\cos \left(\angle\left[\vec{p}_{3}, \vec{p}_{4}\right]\right)
\end{aligned}
$$

where $p_{i}$ are the energy-ordered four-momenta $\left(E_{1}>E_{2}>\right.$ $\left.E_{3}>E_{4}\right)$. The theoretical NLO expression for each of the angular observables is written as

$$
\begin{aligned}
& \frac{1}{\sigma_{4}} \frac{\mathrm{d} \sigma}{\mathrm{d} \cos X}(\cos X) \\
& =\left\{\left(\eta(\mu)^{2} B_{\cos X}(\cos X)+\eta(\mu)^{3}\right.\right. \\
& \left.\quad \times\left[B_{\cos X}(\cos X) \beta_{0} \ln \left(x_{\mu}^{2}\right)+C_{\cos X}(\cos X)\right]\right) \\
& \left./\left(\eta(\mu)^{2} \mathcal{B}_{\cos X}+\eta(\mu)^{3}\left[\mathcal{B}_{\cos X} \beta_{0} \ln \left(x_{\mu}^{2}\right)+\mathcal{C}_{\cos X}\right]\right)\right\}
\end{aligned}
$$

where $\mathcal{B}$ and $\mathcal{C}$ are obtained by integrating the functions $B$ and $C$ over the fit range.

\section{ALEPH detector}

The ALEPH detector is described in detail elsewhere [20]. Briefly, at the core of the tracking system there is a silicon strip vertex detector (VDET). This has two layers providing measurements in both the $r$ - $\phi$ and $r$ - $z$ projections. The spatial resolution for $r-\phi$ coordinates is $12 \mu \mathrm{m}$ for normal incidence and varies between 12 and $22 \mu \mathrm{m}$ for $z$ coordinates. The angular coverage of the VDET is $|\cos \theta|<0.85$ for the inner layer and $|\cos \theta|<0.69$ for the outer layer. The VDET lies within a cylindrical drift chamber (ITC), which measures up to eight coordinates 
per track in the $r-\phi$ projection, with a resolution of 150 $\mu \mathrm{m}$. The ITC is in turn enclosed in a large time projection chamber (TPC), which provides up to 21 threedimensional coordinates per track, with resolutions in the $r-\phi$ and $r-z$ projections of $180 \mu \mathrm{m}$ and $500 \mu \mathrm{m}$, respectively. The three tracking detectors are surrounded by a superconducting solenoid producing a magnetic field of 1.5 T. For tracks with two VDET coordinates, a transverse momentum resolution of $\Delta p_{\mathrm{T}} / p_{\mathrm{T}}=6 \times 10^{-4} p_{\mathrm{T}} \oplus 0.005$ $\left(p_{\mathrm{T}}\right.$ in $\left.\mathrm{GeV} / c\right)$ is achieved.

An electromagnetic calorimeter (ECAL) and a hadron calorimeter (HCAL) are used to measure the energies of neutral and charged particles over almost the full $4 \pi$ solid angle. The ECAL is a lead/wire-chamber sandwich operated in proportional mode and is read out via projective towers subtending typically $0.9^{\circ} \times 0.9^{\circ}$. A relative energy resolution of $0.18 / \sqrt{E}(E$ in $\mathrm{GeV})$ is obtained for isolated showers. The HCAL uses the iron return yoke as an absorber, for a total of 7.5 hadronic interaction lengths. Hadronic showers are sampled by 23 planes of streamer tubes, which induce an analog signal on pads arranged in projective towers of approximately $3.7^{\circ} \times 3.7^{\circ}$. The HCAL provides a measurement of the energy of hadrons with a relative resolution of $0.85 / \sqrt{E}(E$ in $\mathrm{GeV})$. Muon chambers consisting of two double layers of streamer tubes surround the HCAL.

Photons can be reconstructed using the ECAL. Electrons are identified with the ECAL and by using the measured energy loss in the TPC. Muons are seen as tracks giving a series of hits on digital readout strips in the HCAL and muon chamber streamer tubes.

Combining the information of all subdetectors, an energy-flow algorithm [21] provides a measurement of the total energy and a list of charged and neutral reconstructed objects, called energy-flow objects, with measured momentum vectors and information on particle type.

\section{Description of the analysis method}

\subsection{Event selection}

Data from 1994 and 1995 are used, taken at and around the $\mathrm{Z}$ peak by the ALEPH detector. In a first step a hadronic event selection is applied. Good tracks are defined as originating close to the interaction point (with transverse impact parameter $\left|d_{0}\right|<2 \mathrm{~cm}$ and longitudinal impact parameter $\left|z_{0}\right|<10 \mathrm{~cm}$ ), having at least $4 \mathrm{TPC}$ hits, a polar angle in the range $18^{\circ}<\theta<162^{\circ}$, and a transverse momentum $p_{\mathrm{T}}>0.2 \mathrm{GeV} / c$.

Neutral energy-flow objects are defined as good if their polar angle with respect to the beam axis is in the range $18^{\circ}<\theta<162^{\circ}$. Events are selected by requiring at least five good tracks and the total energy $E_{\mathrm{ch}}$ carried by all good tracks to exceed $15 \mathrm{GeV}$. Only events with $\left|\cos \Theta_{\mathrm{Sph}}\right|<0.9$ are kept, where $\Theta_{\mathrm{Sph}}$ is the polar angle of the sphericity axis, computed from all good charged and neutral objects as obtained from the energy-flow algorithm. According to the MC simulation, this basic hadronic event selection is about $90.2 \%$ efficient. Nonhadronic background, which is dominated by $\tau^{+} \tau^{-}$events, represents about $0.3 \%$ of this sample. After the selection a sample of 2.5 million hadronic events remains for further analysis.

All selected charged and neutral energy-flow objects from selected hadronic events are clustered into jets by means of the Durham clustering algorithm, with the E recombination scheme. The metric

$$
y_{i j}=\frac{2 \min \left(E_{i}^{2}, E_{j}^{2}\right)}{E_{\mathrm{vis}}^{2}}\left(1-\cos \theta_{i j}\right)
$$

is used, i.e. those particles ( $i$ and $j$ ) with the smallest $y_{i j}$ are clustered together to form a new pseudo-particle with four-momentum

$$
E_{\text {new }}=E_{i}+E_{j} \quad, \quad \vec{p}_{\text {new }}=\vec{p}_{i}+\vec{p}_{j} .
$$

The clustering is repeated until the minimum $y_{i j}$ is larger than $y_{\text {cut }}$. This procedure is used to calculate the fourjet rate at each $y_{\text {cut }}$ value. In order to avoid high energy photons from final state radiation off quarks, the fraction of electromagnetic energy in each jet is required to be smaller than $90 \%$.

The energies of the jets are rescaled by imposing total energy-momentum conservation keeping the four-jet directions fixed. The Durham metric is recalculated and the event is taken for the calculation of the angular observables if $\min _{i j=1,4} y_{i j}>y_{\text {cut }}$, with $y_{\text {cut }}=0.008$ and $i, j$ running over the four jets. For this resolution parameter about 163,000 events are selected. This choice of $y_{\text {cut }}$ is motivated by the fact that it allows to minimize the two-, three-, five- and six-jet backgrounds while keeping enough statistics and the four jets well separated. In addition, the same value has been used in previous analyses [3,22], thus a comparison of the results is facilitated.

The analyses also use 5.3 million simulated hadronic events produced with a generator based on the JETSET 7.4 parton shower model [23]. The production rates, decay modes and lifetimes of heavy hadrons are adjusted to agree with recent measurements, while heavy quarks are fragmented using the Peterson et al. model [24]. Detector effects are simulated using the GEANT package [25].

\subsection{Hadronization corrections}

The theoretical predictions at parton level have to be corrected in order to take into account hadronization as well as detector effects before being compared to data. The analysis is based on the assumption that hadronization corrections are independent on $\alpha_{s}$ and the colour factors. The hadronization corrections are implemented separately for the four-jet rate and the four-jet angular correlations as detailed below.

\subsubsection{Corrections for $R_{4}$}

For the four-jet rate the hadronization corrections are computed from MC simulations using $R_{4}$ distributions at 
parton and at hadron level. Here, the parton level refers to the set of partons present after the showering process. The correction factors for each $y_{\text {cut }}$ are computed according to

$$
C^{\text {had }}\left(y_{\text {cut }}\right)=\frac{R_{4}^{\text {had }}\left(y_{\text {cut }}\right)}{R_{4}^{\text {part }}\left(y_{\text {cut }}\right)},
$$

where the superscript "had" ("part") refers to the hadron (parton) level.

The JETSET parton shower model together with the Lund string fragmentation scheme (PYTHIA 6.1) is employed for the calculation of the hadronization corrections. The model parameters are taken from [26], with the exception that final state radiation is not included in the simulation. Effects of final state radiation are considered together with detector effects as is explained in the next section. A similar approach for the description of the parton level is taken by the HERWIG 6.1 program [27], which is also used to compute the hadronization corrections. In this case, the fragmentation is modelled according to the cluster fragmentation scheme.

Another approach is tested by using the matrix element (ME) option in PYTHIA. In the ME option (PYTHIA,ME) at the parton level two-, three- and fourparton final states are generated according to the exact NLO matrix elements, and then the hadronization step is performed via the string fragmentation scheme. This model should give a better description of four-jet related quantities. However, it is known not to describe well the energy evolution of basic quantities such as the charged particle multiplicity [28]. A special PYTHIA production which has on average four partons after the parton shower is also tested (PYTHIA, $\left.Q_{0}\right)$. The parton shower cut-off parameter, $Q_{0}$, is increased to $4 \mathrm{GeV}$, and afterwards the fragmentation parameters are retuned so that the hadron level describes the data.

\subsubsection{Corrections for the angular observables}

For the angular observables a different MC simulation is used. In PYTHIA 6.1 the option to start a parton shower from a four-parton configuration is chosen [29]. This MC simulation should better describe the data provided that two- and three-jet backgrounds are negligible, and that the showering and hadronization processes are well modelled. The parameters for the showering and hadronization are identical to the simulation used for the four-jet rate.

An important parameter in this four-parton MC simulation is the so called intrinsic resolution parameter $y_{\text {int }}$. The rejection of four-parton configurations with a $y_{34}$ (resolution parameter when going from four to three jets) smaller than $y_{\text {int }}$ is used to avoid soft and collinear divergences. The parameter $y_{\text {int }}$ has to be smaller than $y_{\text {cut }}$, but going to very small values is not possible for technical reasons. Therefore it is not a suitable $\mathrm{MC}$ for the fourjet rate, which is calculated at different $y_{\text {cut }}$ values over a large range. The value chosen for the MC simulation used to correct the angular distributions is $y_{\text {int }}=0.004$.
With this four-parton option 15 million events were generated with about 8 million four-jet events selected at $y_{\text {cut }}=0.008$. The angular distributions are calculated at three levels: parton level before showering (i.e. using massless LO matrix elements), parton level after showering and hadron level. In order to correct not only for the hadronization effects, but also for higher orders contributions, the bin-by-bin ratios of the distribution at hadron level over the parton level are computed,

$$
C^{\text {had }}\left(i_{\text {bin }}\right)=\frac{\cos X^{\text {had }}\left(i_{\text {bin }}\right)}{\cos X^{\text {part }}\left(i_{\text {bin }}\right)},
$$

where now "part" refers to the parton level before showering and $i_{\text {bin }}$ goes from bin 1 to 20 of the corresponding observable.

The simulation of massless four-parton configurations is also possible using the HERWIG 6.1 MC program. About 6 million events were produced, with about 2.5 million events selected as four-jet events.

The ME option in PYTHIA, as was described for the four-jet rate in Sect. 4.2.1, is also tested for the corrections of the angular distributions. Finally, a fourth MC set was produced with FOURJPHACT [30] in order to check for mass effects. There, the massive LO four-parton MEs are employed for generating the initial state. The showering and hadronization processes are modelled using PYTHIA 6.1 (standard parameters).

\subsection{Detector corrections}

The theoretical predictions, which are corrected to hadron level, have to be corrected further for detector effects such as acceptance, efficiency and resolution before being fitted to data. This is achieved by computing the observables from a MC before and after the detector simulation and imposing the same track and event selection criteria as for the data. Then the correction factors are defined as

$$
C^{\text {det }}\left(i_{\text {bin }} / y_{\text {cut }}\right)=\frac{O_{4}^{\text {det }}\left(i_{\text {bin }} / y_{\text {cut }}\right)}{O_{4}^{\text {had }}\left(i_{\text {bin }} / y_{\text {cut }}\right)}
$$

where $O_{4}^{\text {det }}\left(i_{\text {bin }} / y_{\text {cut }}\right)$ denotes the value of the observable at the detector level. The hadron level distributions are obtained by switching off any photon radiation in the initial and final state (ISR, FSR), both present at the detector level, with all particles having mean lifetimes less than $10^{-9}$ s required to decay, and all other particles being treated as stable. The detector level distributions are obtained with the MC simulation described in Sect.4.1. Typically, the detector correction factors are found within the $5 \%$ range (i.e. corrections with values between 0.95 and $1.05)$, except at the edges of the phase space where corrections up to $10-20 \%$ are observed (i.e. corrections within $0.9 / 0.8$ and $1.1 / 1.2)$.

Another approach is tested for the correction of the angular observables. A detector level distribution is obtained by passing through the detector simulation events 
ALEPH
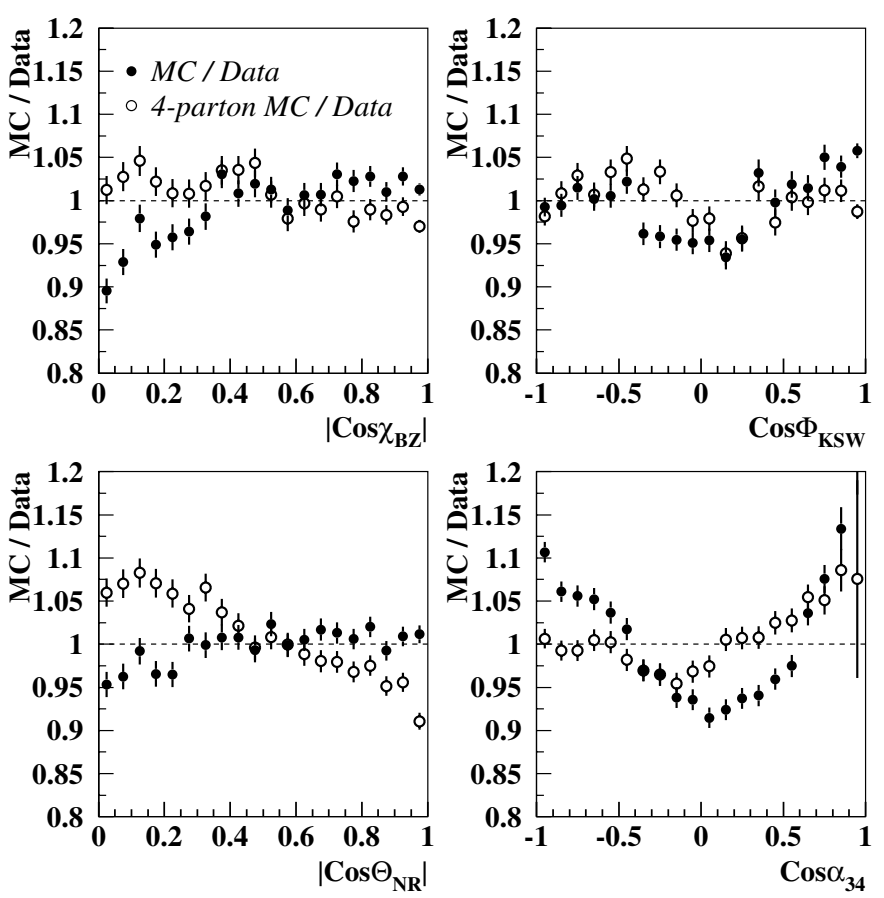

Fig. 1. Comparison of the two sets of MC simulations with respect to ALEPH data at detector level. The 4-parton MC refers to events generated with the PYTHIA option to start a parton shower from a four-parton configuration, passed through detector simulation

simulated with the PYTHIA four-parton option, including ISR and FSR. This MC simulation is expected to describe the data better, which is indeed found for $\cos \chi_{B Z}$ or $\cos \alpha_{34}$, but not for $\cos \Theta_{\mathrm{NR}}$ as shown in Fig. 1. Therefore, this new MC simulation is used to calculate again the correction factors $C^{\text {det }}$ for $\cos \chi_{B Z}$ and $\cos \alpha_{34}$.

\subsection{Fit procedure}

The measured binned distributions for the four-jet angular correlations and the four-jet rate are combined to form a vector

$$
\begin{aligned}
D_{(1 \ldots 140)}=( & \cos \chi_{\mathrm{BZ}_{(1 \ldots 20)}}, \cos \phi_{\mathrm{KSW}_{(1 \ldots 20)}}, \cos \Theta_{\mathrm{NR}_{(1 \ldots 20)}}, \\
& \left.\cos \alpha_{34_{(1 \ldots 20)}}, R_{4_{(1 \ldots 60)}}\right) .
\end{aligned}
$$

The subscripts $1 \ldots 20$ correspond to the twenty equallysized bins used for each angular observable, in case of $R_{4}$ 60 equidistant points, in the range $-12 \leq \ln \left(y_{\text {cut }}\right) \leq-0.2$, are used. The binning of the angular distributions is the same as in previous analyses $[3,22]$, therefore allowing again for an easy comparison of the results.

A covariance matrix $\sigma_{i j}^{D}$ is calculated from data distributions to take into account the statistical error, correlations between bins of a single distribution, and correlations between bins of different distributions. The covariance matrices of the angular distributions are calculated taking into account that they are of multinomial type. The terms in the covariance matrix for bins of different angular observables as well as for the various bins of $R_{4}$ are calculated as

$$
\begin{aligned}
V W_{i j} & =\left((p p)_{i j}-p_{i} p_{j}\right) \frac{1}{N_{\mathrm{had}}}, \\
p_{i} & =\frac{N_{i}}{N_{\mathrm{had}} \Delta}, \\
(p p)_{i j} & =\frac{N_{i j}}{N_{\mathrm{had}} \Delta^{2}}
\end{aligned}
$$

where $N_{i j}$ represents the two-dimensional scatter plot of any two distributions $\mathrm{V}$ and $\mathrm{W}$ and $\Delta$ is the bin width.

Then a further vector is formed, $T_{1 \ldots 140}$, with the theoretical distributions corrected to detector level. With all these inputs, the following function is computed and minimized with respect to the fitted parameters:

$$
\begin{aligned}
\chi^{2} & =\sum_{i, j \in \text { fit range }} \delta_{i} \sigma_{i j}^{-1} \delta_{j}, \\
\delta_{i} & =D_{i}-T_{i}, \\
\sigma_{i j} & =\sigma_{i j}^{D}+\sigma_{i j}^{T},
\end{aligned}
$$

where $\sigma_{i j}^{T}$ takes into account the statistical uncertainties coming from the theoretical distributions as well as from hadronization and detector corrections.

\subsection{Systematic uncertainty studies}

Systematic uncertainties can arise from imperfections of the implementation of the physics processes in the MC as well as from deficiencies in the description of the detector performance, from theoretical uncertainties or missing higher orders in the perturbative series, from the model used to calculate the hadronization corrections, and from the specific analysis procedure. Sources of systematic uncertainties are checked for by studying the fit range, the selection cuts, the hadronization and detector corrections, the scale uncertainty and the mass effects.

A method inspired by Bayesian statistics is used [31] in order to obtain the systematic error for each source, except for the variation of the fit range, where the number of fitted bins is different. The Bayesian idea is that a priori all models can be considered equally well suited for usage in the analysis, but from a bad $\chi^{2}$ in the fit it is deduced that the a posteriori probability of such a model is low, and therefore this model should get a small weight when estimating the actual systematic error.

For example, a measurement of two quantities $(a, b)$ results in a set of numbers $\left(a_{0}, b_{0}, \chi_{0}^{2}\right),\left(a_{1}, b_{1}, \chi_{1}^{2}\right), \ldots,\left(a_{n}, b_{n}\right.$, $\left.\chi_{n}^{2}\right)$ after $(n+1)$ variations of the analysis procedure, with $\chi_{0}^{2}=\min _{i=0, n} \chi_{i}^{2}$. First a correlation coefficient for the systematic errors is calculated according to

$$
\rho_{a, b}^{\mathrm{sys}}=\frac{\sum_{i=1}^{n}\left(a_{0}-a_{i}\right)\left(b_{0}-b_{i}\right)}{\sqrt{\left(\sum_{i=1}^{n}\left(a_{0}-a_{i}\right)^{2}\right)\left(\sum_{i=1}^{n}\left(b_{0}-b_{i}\right)^{2}\right)}} .
$$


Table 1. Fit results with statistical errors only for Methods I to III using ALEPH data

\begin{tabular}{lcccc}
\hline & $\eta\left(M_{\mathrm{Z}}\right)$ & $x_{\mu}$ & $\chi^{2} / N_{\text {dof }}$ & $\alpha_{s}\left(M_{\mathrm{Z}}\right)$ \\
\hline Method I & $0.02483 \pm 0.00003$ & 1. & $27.6 / 5$ & $0.1170 \pm 0.0001$ \\
Method II & $0.02494 \pm 0.00004$ & $0.73 \pm 0.05$ & $4.8 / 4$ & $0.1175 \pm 0.0002$ \\
Method III & $0.02494 \pm 0.00003$ & 0.73 & $4.8 / 5$ & $0.1175 \pm 0.0001$ \\
\hline
\end{tabular}

Then the elements of the systematic covariance matrix are defined as

$$
\begin{aligned}
\sqrt{\sigma_{a, a}^{\mathrm{sys}}} & =C \max _{i=1, n}\left(\frac{\Delta a_{i}}{\sqrt{\Delta \chi^{2}}}\right), \\
\sigma_{a, b}^{\mathrm{sys}} & =\rho_{a, b}^{\mathrm{sys}} \sqrt{\sigma_{a, a}^{\mathrm{sys}}} \sqrt{\sigma_{b, b}^{\mathrm{sys}}},
\end{aligned}
$$

with

$$
\begin{aligned}
\Delta a_{i} & =\left|a_{0}-a_{i}\right| \\
\Delta \chi^{2} & =\max \left(1,\left|\chi_{0}^{2}-\chi_{i}^{2}\right|\right) \\
C & =\max \left(1, \sqrt{\chi_{0}^{2} / N_{\text {dof }}}\right) .
\end{aligned}
$$

The factor $C$ takes into account cases where even the best fit gives a bad $\chi^{2}$. This scheme is generalizable to any number of fit variables, and it is ensured that models giving a bad fit are properly deweighted. Of course still some unavoidable arbitrariness remains in the choice and number of variations.

\section{Measurements of the strong coupling constant from the four-jet rate}

\subsection{Corrections for the four-jet rate}

The bin-by-bin hadronization corrections calculated with the various models described in Sect.4.2.1 are computed. The two parton shower models PYTHIA and HERWIG give very similar corrections, which differ appreciably from unity, by about $20 \%$. The corrections obtained with the PYTHIA,ME and PYTHIA, $Q_{0}$ options typically deviate even more from unity and are quite different from the standard corrections. The large discrepancies at the order of $10 \%$ can be traced back to large discrepancies in the four-jet rates at parton and at hadron level. The parton shower option, for $y_{\text {cut }}=0.008$, gives a four-jet rate of $8.2 \%(6.9 \%)$ at parton (hadron) level, whereas the matrix element option predicts $10.2 \%(7.7 \%)$.

Taking into account the hadronization and detector corrections, as discussed in Sect. 4.3, the total corrections for the four-jet rate can be constructed as:

$$
C^{\text {tot }}\left(y_{\text {cut }}\right)=C^{\text {had }}\left(y_{\text {cut }}\right) \cdot C^{\text {det }}\left(y_{\text {cut }}\right) .
$$

Figure 2 shows the total bin-by-bin corrections. They amount to about $10 \%$ in the central region of the fourjet rate, but increase rapidly to around $20 \%$ or higher

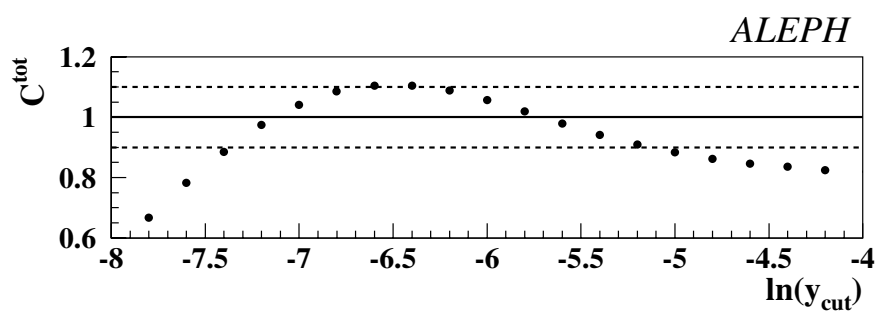

Fig. 2. Total corrections for the four-jet rate. The dashed lines show the maximum allowed corrections used for the fit

when going to small or large $y_{\text {cut }}$ values. The fit range is required to be within $-6.4<\ln \left(y_{\text {cut }}\right)<-5.4$, where the total correction is smaller than $10 \%$ and non-perturbative effects are expected to be small.

\subsection{Results}

A $\chi^{2}$ is constructed according to (16), where $i$ and $j$ run over the bins allowed by the fit range requirements. Three different minimizations of this $\chi^{2}$ are carried out, leading to three different measurements of the strong coupling constant.

- Method I. The minimization is performed with respect to $\eta=\frac{\alpha_{s} C_{F}}{2 \pi}$, with $x_{\mu}$ fixed to 1 . Later, all the systematic uncertainty estimations are done at $x_{\mu}=1$. The scale uncertainty is estimated as the variation in the fitted $\eta$ when $x_{\mu}$ is varied in the range $0.5<x_{\mu}<$ 2.

- Method II. The minimization is performed with respect to $\eta$ and $x_{\mu}$. In all the systematic uncertainty estimations both parameters are fitted again. There is no theoretical uncertainty associated to the scale, as it is a fitted parameter.

- Method III. The minimization is first performed with respect to both $\eta$ and $x_{\mu}$. The fitted $x_{\mu}$ value is taken as the optimized scale, $x_{\mu}^{\text {opt }}$. Then, all the systematic uncertainty estimations are calculated by fitting only $\eta$, but with the scale fixed to this optimized value. The scale uncertainty is estimated by the variation in $\eta$ when $x_{\mu}$ is moved in the range $0.5 x_{\mu}^{\text {opt }}<x_{\mu}<2 x_{\mu}^{\text {opt }}$.

The fit results for the three methods can be found in Table 1 and the plots corresponding to the best fits in Fig. 3.

A $\chi^{2}$ per degree of freedom close to unity is found for methods II and III, where a "preferred" value $x_{\mu}=0.73$ 

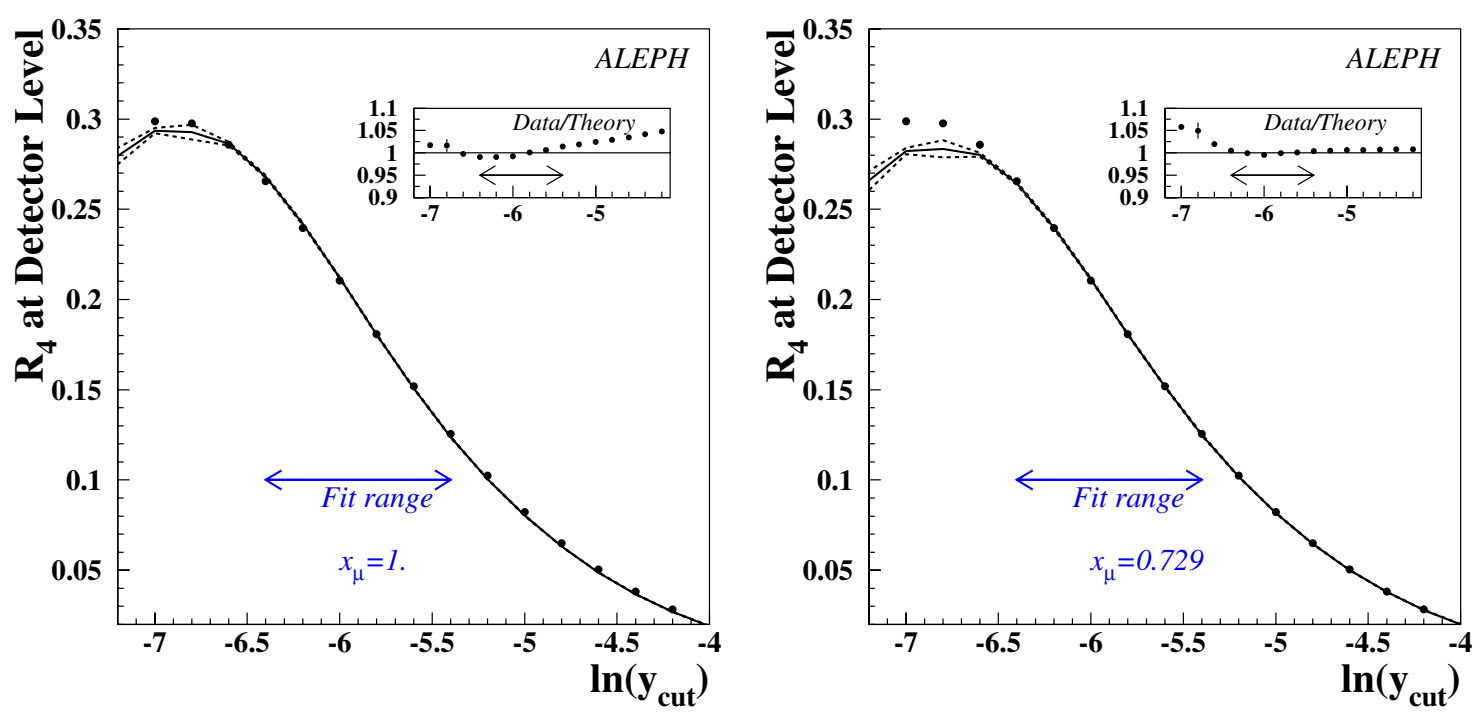

Fig. 3. Distribution of the four-jet rate, corrected to detector level and fitted to ALEPH data using Method I (left) and Method II/III (right). Dots correspond to ALEPH data and the solid line to the fitted distribution. Dashed lines indicate the statistical uncertainty. The ratio of data with respect to fitted distributions is shown in the small insert

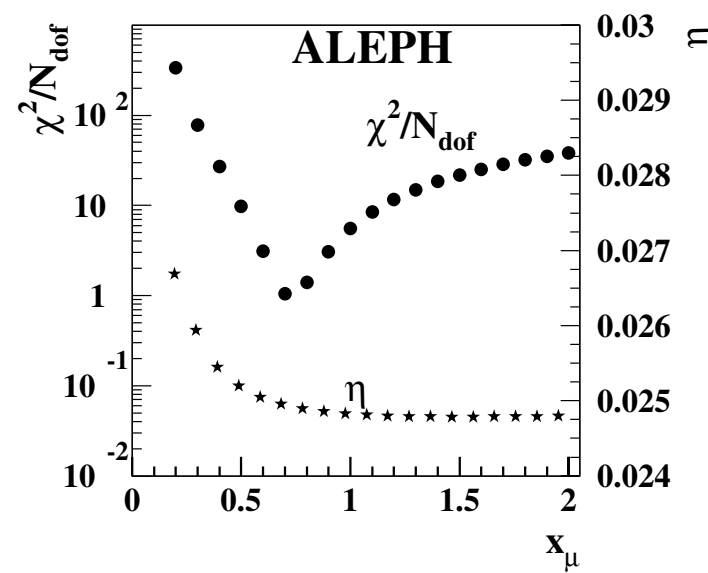

Fig. 4. Dependence of the fit results for $\eta$ and $\chi^{2} / N_{\text {dof }}$ on the renormalization scale $x_{\mu}$

is obtained. The fit result for $x_{\mu}$ in Method II, different from unity, might be an indication that missing higher orders in perturbative QCD are still important. This is also reflected in the large $\chi^{2}$ for Method I, where the scale is not allowed to vary and thus to mimic the contributions from missing higher orders. In Fig. 3 it is illustrated that the fitted four-jet rate deviates significantly from data for low values of $\ln y_{\text {cut }}$ outside of the fit range.

Finally, Fig. 4 shows the sensitivity of the fit to the renormalization scale, leading to a theoretical uncertainty on $\eta$ from the scale variation.

\subsection{Systematic studies}

Tables $2-4$ show the sources of systematic uncertainty that are studied for the three methods. A description of each uncertainty source is found below.
Table 2. Systematic uncertainties for Method I

\begin{tabular}{lcc}
\hline & $\eta\left(M_{\mathrm{Z}}\right)$ & $\chi^{2} / N_{\text {dof }}$ \\
\hline tot.corr. $<20 \%$ & $0.02491 \pm 0.00002$ & $57.5 / 8$ \\
tot.corr. $<5 \%$ & $0.02480 \pm 0.00003$ & $6.1 / 1$ \\
\hline range sys. & $\Delta \eta=0.00008$ & \\
\hline charged only & $0.02500 \pm 0.00003$ & $33.6 / 5$ \\
experimental sys. & $\Delta \eta=0.00016$ & \\
\hline HERWIG & $0.02461 \pm 0.00003$ & $107.3 / 5$ \\
hadr. sys. & $\Delta \eta=0.00006$ & \\
\hline$x_{\mu}=0.5$ & $0.02519 \pm 0.00003$ & $50.0 / 5$ \\
$x_{\mu}=2$. & $0.02480 \pm 0.00002$ & $195.3 / 5$ \\
scale sys. & $\Delta \eta=0.00018$ & \\
only light flavours & $0.02476 \pm 0.00004$ & $17.8 / 5$ \\
mass sys. & $\Delta \eta=0.00005$ & \\
total theoretical sys. & $\Delta \eta=0.00019$ & \\
\hline
\end{tabular}

Table 3. Systematic uncertainties for Method II

\begin{tabular}{lccc}
\hline & $\eta\left(M_{\mathrm{Z}}\right)$ & $x_{\mu}$ & $\chi^{2} / N_{\text {dof }}$ \\
\hline tot.corr. $<20 \%$ & $0.02496 \pm 0.00003$ & $0.756 \pm 0.034$ & $20.3 / 7$ \\
range sys. & $\Delta \eta=0.00002$ & $\Delta x_{\mu}=0.027$ & \\
\hline charged only & $0.02511 \pm 0.00004$ & $0.731 \pm 0.046$ & $9.1 / 4$ \\
experimental sys. & $\Delta \eta=0.00009$ & $\Delta x_{\mu}=0.001$ & \\
\hline HERWIG & $0.02491 \pm 0.00005$ & $0.547 \pm 0.029$ & $0.7 / 4$ \\
hadr. sys. & $\Delta \eta=0.00002$ & $\Delta x_{\mu}=0.099$ & \\
\hline only light flavours & $0.02471 \pm 0.00004$ & $1.575 \pm 0.251$ & $4.6 / 4$ \\
mass sys. & $\Delta \eta=0.00025$ & $\Delta x_{\mu}=0.933$ & \\
\hline
\end{tabular}

\subsubsection{Fit range}

The sensitivity to the fit range is checked by repeating the analyses with the requirement of a total correction per bin smaller than $20 \%$ and $5 \%$ for Method I, and smaller 
Table 4. Systematic uncertainties for Method III

\begin{tabular}{lcc}
\hline & $\eta\left(M_{\mathrm{Z}}\right)$ & $\chi^{2} / N_{\text {dof }}$ \\
\hline tot.corr. $<20 \%$ & $0.02497 \pm 0.00003$ & $20.1 / 8$ \\
range sys. & $\Delta \eta=0.00003$ & \\
\hline charged only & $0.02511 \pm 0.00003$ & $9.1 / 5$ \\
experimental sys. & $\Delta \eta=0.00008$ & \\
\hline HERWIG & $0.02473 \pm 0.00003$ & $27.9 / 5$ \\
hadr. sys. & $\Delta \eta=0.00004$ & \\
\hline$x_{\mu}=0.365$ & $0.02559 \pm 0.00004$ & $193.3 / 5$ \\
$x_{\mu}=1.458$ & $0.02479 \pm 0.00003$ & $101.8 / 5$ \\
scale sys. & $\Delta \eta=0.00005$ & \\
only light flavours & $0.02487 \pm 0.00004$ & $50.8 / 5$ \\
mass sys. & $\Delta \eta=0.00001$ & \\
total theoretical sys. & $\Delta \eta=0.00005$ & \\
\hline
\end{tabular}

than $20 \%$ for Methods II and III. The second variation cannot be used in the last two methods as only two points are selected and the fit of $\eta$ together with $x_{\mu}$ is unstable. The resulting systematic variations due to these new fit ranges are quite different for the three cases. For the first method the range uncertainty is large with respect to the statistical error, whereas for the two other methods is of the same order.

\subsubsection{Experimental uncertainties}

All cuts imposed in the hadronic selection are varied in order to evaluate the effect on the measurement. The new values for the selection cuts on track parameters are found by changing them until the number of selected events per unit luminosity is the same in data and MC. The analysis is repeated by introducing the following changes (only one at a time): at least six measured space coordinates from the TPC; a polar angle in the range $20^{\circ}<\theta<160^{\circ}$ both for charged and neutral tracks; transverse momentum $p_{T}>0.205 \mathrm{GeV} / c ; d_{0}=1.867 \mathrm{~cm} ; z_{0}=6.64 \mathrm{~cm}$; at least 8 selected charged tracks; minimum charged energy $22 \mathrm{GeV} ;\left|\cos \Theta_{\mathrm{Sph}}\right|<0.85$; and fraction of electromagnetic energy $<20 \%$.

The observed changes when modifying the selection cuts are in all cases negligible. These uncertainties are smaller than the equivalent ones obtained with three-jet observables, as might be expected from the quadratic LO dependence on $\eta$ for four-jet variables instead of the linear dependence in the case of three-jet variables.

An estimation of the systematic uncertainty due to the detector corrections has been obtained by repeating the analysis using charged particle tracks only, leading to a variation in $\eta$ going from $0.6 \%$ to $0.3 \%$, depending on the method.

\subsubsection{Hadronization corrections}

The hadronization uncertainty is taken as the change in $\eta$ (using the Bayesian method) when the corrections are calculated with HERWIG. This results in a systematic uncertainty much smaller than $1 \%$ for the three methods. In methods I and III the $\chi^{2}$ of the fit when using HERWIG corrections is almost four times larger than in the standard measurement. Therefore the uncertainty calculated using the Bayesian method is heavily reduced if compared to the full difference between the fit results when using PYTHIA or HERWIG. However, even if the full difference is taken as an estimate of the hadronization uncertainty, it is smaller than 1\% (0.00022).

\subsubsection{Theoretical predictions}

The lack of knowledge of higher orders of perturbative QCD is estimated by the impact on $\eta$ of the renormalization scale variation for Methods I and III.

An estimate of the effect of using massless theoretical predictions for the four-jet rate is performed. First new hadronization corrections are calculated following Sect. 4.2.1, but using parton and hadron level distributions with $\mathrm{u}$ and d quarks only. New detector corrections are calculated by using the hadron level distribution with $\mathrm{u}$ and d quarks only, and a detector level distribution based on a simulation including five flavours, but with the following anti-btag applied, $-\log _{10}\left(P_{\text {uds }}\right)<0.5$. Here $P_{\text {uds }}$ gives the probability that all tracks originate from the main vertex [32]. This selects hadronic events enriched in light quarks with an efficiency of $66 \%$ and a purity of $88 \%$. The same anti-btag is applied to 1994 ALEPH data. Finally, the resummed NLO prediction for the four-jet rate is corrected to detector level using these massless hadronization and detector corrections and fitted to the data distribution. The resulting uncertainties due to mass effects are 0.00005 for Method I, 0.00025 for Method II and 0.00001 for Method III.

The scale uncertainty is the largest contribution to the total systematic uncertainty in the case of Method I as can be seen in Table 2. For Method III, this uncertainty is heavily reduced to less than $1 / 3$ of its value in the first case. This is a well known feature of the optimized scale method [33]. For Method II the mass uncertainty is the largest.

The theoretical uncertainty is estimated as the quadratic sum of the scale and the mass uncertainty for Methods I and III and is equal to the mass uncertainty for Method II, since there is no scale uncertainty.

\subsection{Further checks}

\subsubsection{Hadronization corrections}

As a cross-check, the last two models presented in Sect. 4.2.1 are used to fit $\eta$. The systematic changes in the fitted parameters are covered by the total uncertainty.

\subsubsection{Scale dependence when using PYTHIA or HERWIG}

In the results for Method III, the fitted scale is found to be quite different when using the hadronization correc- 
tions coming from PYTHIA (0.73) or HERWIG (0.55). In order to understand the origin of such a difference the fit is repeated, for some arbitrarily chosen ranges, using corrections from both MC programs. The discrepancies in the fitted $x_{\mu}$ are found to be larger when going to small values of $\ln \left(y_{\text {cut }}\right)$, where the PYTHIA and HERWIG corrections differ more from each other. In fact, for the fit ranges where the corrections from both models are similar, also the fit results for $x_{\mu}$ are compatible within errors. Therefore the difference in $x_{\mu}$ when using PYTHIA or HERWIG corrections is just a propagation of the discrepancies of the hadronization corrections and as such covered by the hadronization plus range uncertainties. In any case, the variations in $\eta$ due to the different ranges considered are small and covered by the total uncertainty.

\subsubsection{Fits over different ranges in $R_{4}$}

Different fits are performed for small ranges covering in total a large region in terms of $\ln \left(y_{\text {cut }}\right)$. The method used for the fit is always Method I. The uncertainty in $\eta, \Delta \eta_{\text {range }}$, is calculated as the largest difference between the measurement at a given range and any of the measurements at other ranges. Then, the scale uncertainty for each range is obtained by varying $x_{\mu}$ from 0.5 to 2 . A large correlation between the range uncertainty and the scale uncertainty is observed, which implies that the "bias" in the fitted $\eta$ which could be introduced by selecting a given range is fully covered by the scale uncertainty in each case, i.e. the fit results for $\eta$ together with their scale uncertainty for the different ranges are fully compatible.

\subsection{Final results}

Combining all systematic uncertainties considered above, the final results of the measurements of the strong coupling constant are:

$$
\begin{gathered}
\eta\left(M_{\mathrm{Z}}\right)=0.02483 \pm 0.00003(\text { stat }) \pm 0.00027(\text { sys }) \\
\Downarrow \\
\alpha_{s}\left(M_{\mathrm{Z}}\right)=0.1170 \pm 0.0001(\text { stat }) \pm 0.0013(\text { sys })
\end{gathered}
$$

for Method I,

$$
\begin{gathered}
\eta\left(M_{\mathrm{Z}}\right)=0.02494 \pm 0.00004(\text { stat }) \pm 0.00027(\text { sys }) \\
\Downarrow \\
\alpha_{s}\left(M_{\mathrm{Z}}\right)=0.1175 \pm 0.0002(\text { stat }) \pm 0.0013(\text { sys })
\end{gathered}
$$

for Method II and, finally for Method III,

$$
\begin{gathered}
\eta\left(M_{\mathrm{Z}}\right)=0.02494 \pm 0.00003(\text { stat }) \pm 0.00011(\text { sys }) \\
\Downarrow \\
\alpha_{s}\left(M_{\mathrm{Z}}\right)=0.1175 \pm 0.0001(\text { stat }) \pm 0.0005(\text { sys })
\end{gathered}
$$

If the Bayesian method is not used, and instead all the contributions for each uncertainty source are added quadratically, the total systematic uncertainty in $\alpha_{s}$ turns out to be $0.0022,0.0014$ and 0.0033 for the three methods. Within the uncertainties the three measurements are consistent with each other. Method I is taken as the final result since it allows a better separation of different components of the systematic uncertainty.

The high precision of this result can be traced back to the remarkable stability of the coupling constant under a variation of the renormalization scale (Fig. 4), and to a general reduction of systematic uncertainties on $\alpha_{s}\left(M_{\mathrm{Z}}\right)$ compared to three-jet quantities because of the leading quadratic dependence of the four-jet rate on the coupling.

\section{A simultaneous measurement of the strong coupling constant and the QCD colour factors}

In this section a new combined measurement of the strong coupling constant and the colour factors using NLO calculations is presented, by fitting the NLO plus resummation predictions for the four-jet rate and the normalized NLO predictions for the four-jet angular correlations to ALEPH data. In the following subsections details about corrections, fit results and systematic uncertainties are given.

\subsection{Corrections for the four-jet observables}

The correction procedure for hadronization is applied, as explained in Sect. 4.2. Large differences are found between the correction factors obtained with HERWIG and PYTHIA. In both cases parameter sets are used which were obtained from a tuning of the standard simulations, namely starting from $q \bar{q}$ configurations. This could be an indication for the non-universality of these parameters.

Taking into account the hadronization and detector corrections, Sect.4.3, the total corrections for each fourjet observable can be constructed as

$$
C^{\text {tot }}\left(i_{\text {bin }}\right)=C^{\text {had }}\left(i_{\text {bin }}\right) \cdot C^{\text {det }}\left(i_{\text {bin }}\right) .
$$

Figure 5 shows the total bin-by-bin correction factors for the angular observables when using PYTHIA for the hadronization corrections. Typically the corrections are found within the $5-10 \%$ range.

\subsection{Results}

A $\chi^{2}$ minimization is performed with respect to $\eta, x$ and $y$, using statistical errors only, and taking into account binto-bin correlations. The fit range is selected by requiring the total corrections for each observable to be smaller than $10 \%$. The results are

$$
\begin{aligned}
\eta\left(M_{\mathrm{Z}}\right) & =0.0255 \pm 0.0003(\text { stat }) \\
x & =2.17 \pm 0.06(\text { stat }) \\
y & =0.37 \pm 0.02(\text { stat })
\end{aligned}
$$


with $\chi^{2} / N_{\text {dof }}=76.8 / 80$ and the correlations $\rho_{\eta x}=-0.85$, $\rho_{\eta y}=-0.45$ and $\rho_{x y}=0.84$. They are in good agreement with both QCD expectations and previous results $[2,3]$. However, an important reduction of the statistical error is achieved.

The fitted distributions are illustrated in Figs. 6 and 7. In the case of $\cos \alpha_{34}$ a discrepancy in the central region of the distribution is observed. This disagreement was already seen in [3]. For the four-jet rate the disagreement between the fitted predictions and the data at small values of $y_{\text {cut }}$ are observed again.

\subsection{Systematic studies}

Table 5 shows the systematic uncertainties taken into consideration. A brief description of each of them is found below.

\subsubsection{Fit range}

The sensitivity to the fit range is checked by repeating the analysis with the requirement of a total correction per bin smaller than $20 \%$ and $5 \%$. In the second case only two points are selected for the four-jet rate. Therefore the fit is repeated but now asking for a total correction per bin smaller than $5 \%$ for the angular distributions and $10 \%$ for $R_{4}$. The systematic variation due to these new fit ranges is smaller than or equal to the statistical errors of the measurement.

\subsubsection{Experimental uncertainty}

All cuts imposed in the selection of hadronic events are moved in order to evaluate the effect on the measurement. The new values for the selection are given in Sect. 5.3.2. The observed changes when modifying the selection cuts are in all cases negligible.

An estimation of the systematic uncertainty due to the detector corrections is obtained by repeating the analysis using charged particle tracks only. This change in the measurement procedure leads to systematic deviations in the parameters of about $1 \%$ for $\eta$ and $x$, and about $3 \%$ for $y$. Another estimate is obtained by means of the four-parton full MC simulation described in Sect. 4.3, which is only used to correct $\cos \chi_{B Z}$ and $\cos \alpha_{34}$. It results in variations similar to using charged particle tracks only. The final uncertainty due to detector corrections is calculated by taking into account the two sources described above. The result is 0.0001 for $\eta$; for the colour factor ratios $\mathrm{x}$ and $\mathrm{y}$ it is 0.02 and 0.01 , respectively.

\subsubsection{Hadronization corrections}

The hadronization uncertainty is taken as the change in the fitted parameters when the corrections are calculated with HERWIG. The values can be found in Table 5 and show large systematic variations, up to $8.5 \%$ for $y$. In this case the $\chi^{2}$ increases substantially.
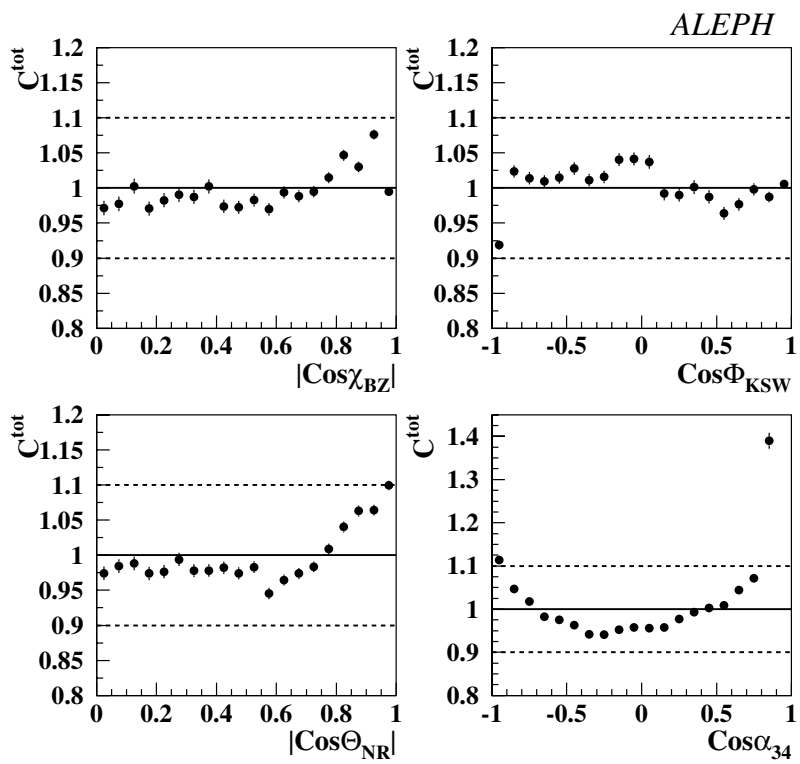

Fig. 5. Total correction factors for the four-jet angular correlations with PYTHIA. The dashed lines show the maximum allowed corrections used for the fit

\subsubsection{Theoretical predictions}

The lack of knowledge of higher order QCD corrections is estimated by varying the renormalization scale in the theoretical predictions. The scale is varied from $x_{\mu}=0.5$ to $x_{\mu}=2$, and the largest difference to the values found for $x_{\mu}=1$ is taken as systematic uncertainty. As the theoretical predictions for $R_{4}$ and for the angular observables are known at different accuracy, the scale uncertainty is estimated by varying $x_{\mu}$ separately for each of the two types of observables. The resulting uncertainty is $4 \%$ for $\eta, 2 \%$ for $x$ and $13 \%$ for $y$. It is the dominant source of uncertainty for all parameters.

The experimentally optimized scale method, applied to the measurement of the strong coupling constant from the four-jet rate, is not suitable for the combined measurement, since different scale dependences are involved. Moreover, the strong correlations between the colour factor ratios and the renormalization scale because of the $\beta$ function (5) would introduce instabilities in the fit.

An evaluation of mass effects for the angular observables, which are not included in the theoretical predictions, is attempted by using the FOURJPHACT program. As the parameters for PYTHIA were optimized for massless partons, the hadronization and background corrections with massive partons for the angular observables are calculated as follows,

$$
C^{\text {had }}\left(i_{\text {bin }}\right)=\frac{\cos X^{\text {part-4j }}\left(i_{\text {bin }}\right)}{\cos X^{\text {part-py }}\left(i_{\text {bin }}\right)} \frac{\cos X^{\text {had-py }}\left(i_{\text {bin }}\right)}{\cos X^{\text {part-py }}\left(i_{\text {bin }}\right)},
$$

where the index part- $4 \mathrm{j}$ indicates the parton level in FOURJPHACT, and part-py (had-py) the parton (hadron) level from PYTHIA. The first ratio corrects for mass effects in the LO prediction, and the second ratio assumes that the showering and hadronization corrections 

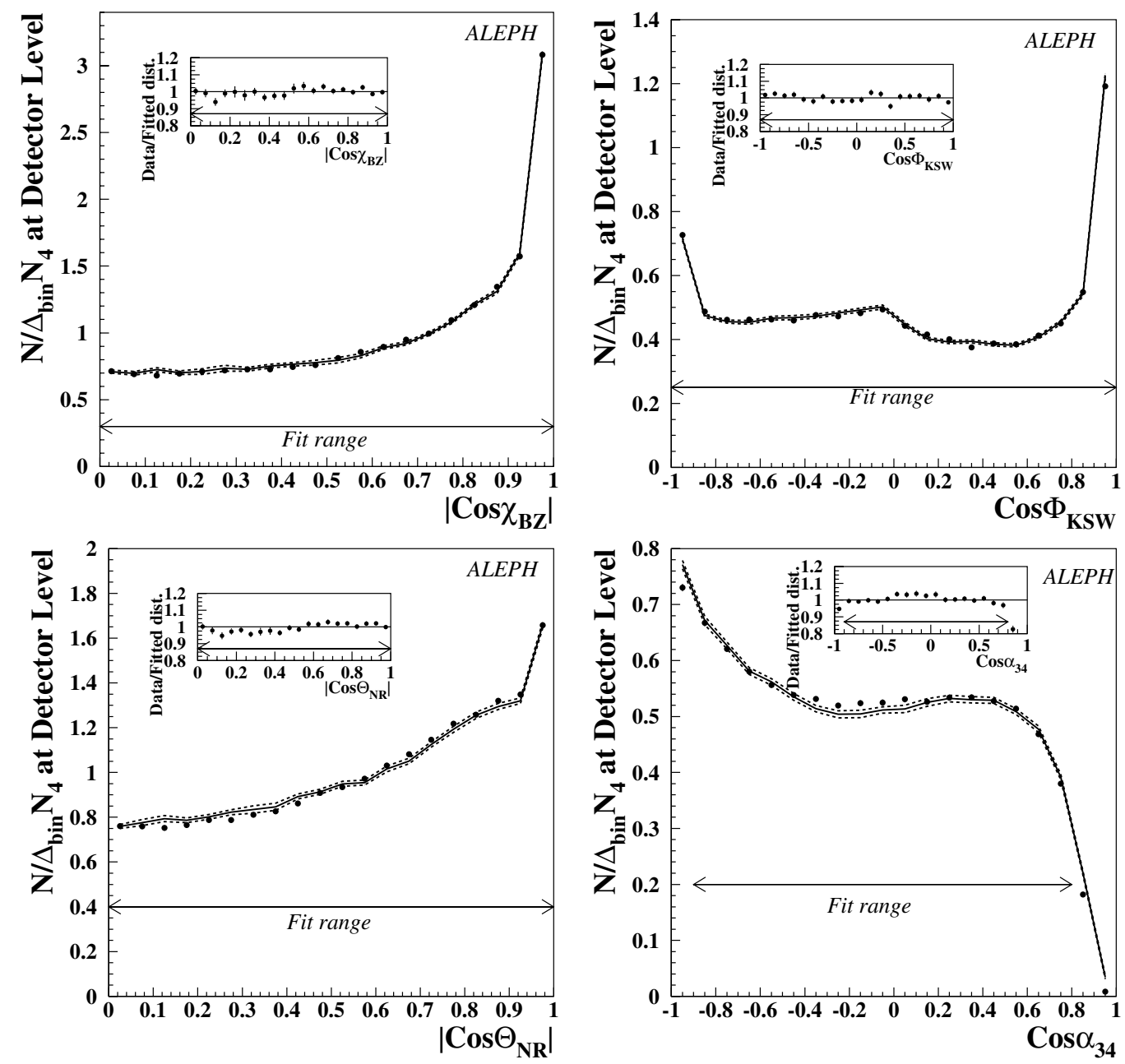

Fig. 6. Comparison of ALEPH data and fit results for the angular correlations in four-jet events. The curves are obtained at detector level. Dots correspond to ALEPH data. The solid lines show the fitted distributions while dashed lines correspond to their statistical uncertainty. The ratio of data with respect to the fitted distributions is shown in the small inserts

do not depend strongly on the quark masses. It is found that mass effects might be large, up to 0.04 for $x$.

Mass effects for the four-jet rate are evaluated in the same manner as explained in Sect. 5.3.4. They are found to be large for the three parameters: 0.0009 for $\eta$ and 0.04 both for $x$ and $y$.

The total mass uncertainty is obtained by adding quadratically the uncertainties in each parameter when considering mass effects in the four-jet rate and in the angular observables. Finally, the total theoretical uncertainty is obtained by adding quadratically the contribution of the two sources described above, i.e. scale and mass uncertainties.

\subsection{Further checks}

\subsubsection{Hadronization corrections}

As a cross-check, the last two models presented in Sect. 4.2.1 are used to fit $\eta$ and the colour factor ratios. The changes in the fitted parameters are of about $2-3 \%$, which is covered by the total uncertainty. Finally, also the standard PYTHIA simulation, namely a parton shower starting from a pair $\mathrm{q} \overline{\mathrm{q}}$, is used to correct the four-jet angular distributions. As expected, the $\chi^{2}$ of the fit is much larger than for the nominal fit, showing that the PYTHIA simulation which uses four-parton matrix elements and a parton shower describes better the shape of correlations in four-jet events.

\subsubsection{Two- and three-parton backgrounds for the four-jet angular correlations}

The hadronization corrections used for the four-jet angular correlations are valid provided that the number of twoand three-parton events that are clustered into four jets after hadronization is negligible. This is verified by the following study.

Using the PYTHIA ME option as described in Sect. 4.2.1, 1 million events are generated, with standard hadronization parameters. The fraction of the number of 
Table 5. Systematic uncertainties due to the various sources considered for the combined fit. $(\mathrm{HW}=\mathrm{HERWIG}, \mathrm{PY}=\mathrm{PYTHIA})$

\begin{tabular}{lcccc}
\hline & $\eta\left(M_{\mathrm{Z}}\right)$ & $x$ & $y$ & $\chi^{2} / N_{\mathrm{dof}}$ \\
\hline tot.corr. $<20 \%$ & $0.02565 \pm 0.00021$ & $2.191 \pm 0.056$ & $0.387 \pm 0.019$ & $89.0 / 88$ \\
tot.corr. $<5 \%$ angles & $0.02545 \pm 0.00031$ & $2.191 \pm 0.065$ & $0.376 \pm 0.020$ & $67.0 / 75$ \\
tot.corr. $<5 \%$ all & $0.02548 \pm 0.00066$ & $2.196 \pm 0.069$ & $0.382 \pm 0.031$ & $63.0 / 71$ \\
range sys. & $\Delta \eta=0.0001$ & $\Delta x=0.03$ & $\Delta y=0.02$ & \\
\hline charged only & $0.02577 \pm 0.00031$ & $2.143 \pm 0.062$ & $0.359 \pm 0.020$ & $82.5 / 80$ \\
4-partons full MC & $0.02584 \pm 0.00031$ & $2.082 \pm 0.061$ & $0.342 \pm 0.020$ & $107.0 / 80$ \\
experimental sys. & $\Delta \eta=0.0001$ & $\Delta x=0.02$ & $\Delta y=0.01$ & \\
\hline HW - all & $0.02592 \pm 0.00033$ & $2.207 \pm 0.072$ & $0.428 \pm 0.023$ & $432 . / 80$ \\
HW - angles, PY - $R_{4}$ & $0.02508 \pm 0.00032$ & $2.225 \pm 0.071$ & $0.370 \pm 0.023$ & $412 . / 80$ \\
PY - angles, HW - $R_{4}$ & $0.02639 \pm 0.00033$ & $2.135 \pm 0.064$ & $0.417 \pm 0.020$ & $79.1 / 80$ \\
hadr. sys. & $\Delta \eta=0.0006$ & $\Delta x=0.02$ & $\Delta y=0.03$ & \\
\hline$x_{\mu}=0.5$ for the angles & $0.02545 \pm 0.00032$ & $2.193 \pm 0.067$ & $0.377 \pm 0.021$ & $64.8 / 80$ \\
$x_{\mu}=2$. for the angles & $0.02558 \pm 0.00030$ & $2.148 \pm 0.059$ & $0.361 \pm 0.019$ & $87.9 / 80$ \\
$x_{\mu}=0.5$ for $R_{4}$ & $0.02352 \pm 0.00030$ & $2.265 \pm 0.062$ & $0.266 \pm 0.018$ & $72.6 / 80$ \\
$x_{\mu}=2$. for $R_{4}$ & $0.02712 \pm 0.00031$ & $2.096 \pm 0.063$ & $0.439 \pm 0.021$ & $86.8 / 80$ \\
scale sys. & $\Delta \eta=0.0010$ & $\Delta x=0.05$ & $\Delta y=0.05$ & \\
mass eff. - angular obs. & $0.02568 \pm 0.00035$ & $2.122 \pm 0.062$ & $0.354 \pm 0.020$ & $75.6 / 80$ \\
mass eff. - $R_{4}$ & $0.02354 \pm 0.00031$ & $2.261 \pm 0.061$ & $0.284 \pm 0.021$ & $71.9 / 80$ \\
mass sys. & $\Delta \eta=0.0009$ & $\Delta x=0.06$ & $\Delta y=0.04$ & \\
theoretical sys. & $\Delta \eta=0.0014$ & $\Delta x=0.09$ & $\Delta y=0.07$ & \\
\hline
\end{tabular}

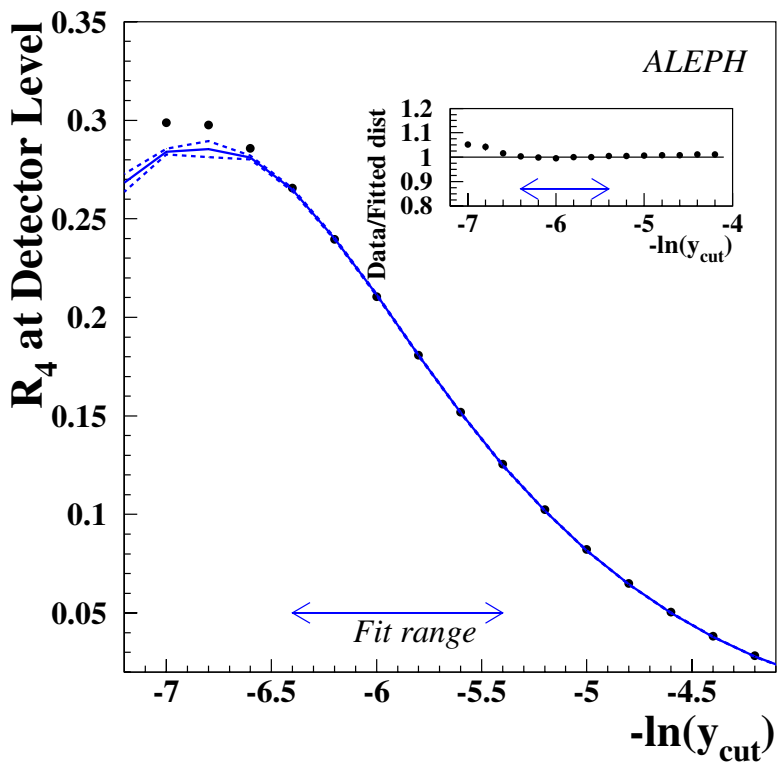

Fig. 7. Comparison of ALEPH data and fit results for the four-jet rate. The curves are obtained at detector level. Dots correspond to ALEPH data and the solid line to the fitted distribution. The statistical uncertainty is indicated by dashed lines. The ratio of data with respect to the fitted distributions is shown in the small insert

four-jet events at hadron level arising from two- and threeparton events with respect to the total number of four-jet events is found to be much smaller than $1 \%$, and only slightly affecting the shape of the angular observables.
In order to quantify how the two- and three-parton backgrounds could bias the measurement, a fit is performed to the four-jet angular correlations obtained at hadron level from the PYTHIA ME simulation. The hadronization corrections are calculated using the PYTHIA four-parton option. Then, the background contributions, i.e. non four-jet configurations, are subtracted and the resulting distributions are fitted again. The difference in the fit results are taken as an estimate of the two- and three-parton background uncertainty. They are found to be much smaller than most of the systematic uncertainties and therefore not considered any longer.

\subsubsection{Sensitivity checks}

The sensitivity of the analysis to each of the observables is studied. The fit procedure is repeated five times, excluding one observable at a time. As expected, $\eta$ is mainly fixed by the four-jet rate, and the colour factor ratios by the angular observables. The sensitivity of the different four-jet angular correlations to the colour factors is quite similar.

\subsubsection{Dependence on $y_{\text {cut }}$}

It is checked if the present measurement depends on the chosen value of $y_{\text {cut }}$. The analysis is repeated with fourjet events found for $y_{\text {cut }}=0.01$, which represents a drop in the four-jet rate from $7.1 \%$ for the nominal $y_{\text {cut }}$ value 




Fig. 8. $68 \%$ confidence level contour in the $\left(x=\frac{C_{A}}{C_{F}}, y=\frac{T_{R}}{C_{F}}\right)$ plane, calculated from statistical plus systematic uncertainties (shaded region). For comparison also the results from previous measurements are given, as well as predictions for simple Lie groups

to $5.4 \%$. The results are in agreement with the standard analysis.

\subsection{Final results}

Combining all systematic uncertainties considered above, the final result of the combined measurement of $\eta$ and the colour factor ratios is:

$$
\begin{array}{cc}
\eta\left(M_{\mathrm{Z}}\right)=0.0255 \pm 0.0003(\text { stat }) \pm 0.0014(\mathrm{sys}) \\
x=2.17 \pm 0.06(\text { stat }) \pm 0.09(\mathrm{sys}) \\
y=0.37 \pm 0.02(\text { stat }) \pm 0.07(\mathrm{sys}) \\
\\
\left(\rho_{\eta x}\right)_{\text {stat }}=-0.85 & \left(\rho_{\eta x}\right)_{\mathrm{sys}}=-0.74 \\
\left(\rho_{\eta y}\right)_{\text {stat }}=-0.45 & \left(\rho_{\eta y}\right)_{\mathrm{sys}}=0.83 \\
\left(\rho_{x y}\right)_{\text {stat }}=0.84 & \left(\rho_{x y}\right)_{\mathrm{sys}}=-0.33
\end{array}
$$

which can also be expressed in terms of the strong coupling constant and the colour factors,

$$
\begin{aligned}
\alpha_{s}\left(M_{\mathrm{Z}}\right) & =0.119 \pm 0.006(\text { stat }) \pm 0.026(\mathrm{sys}) \\
C_{A} & =2.93 \pm 0.14(\text { stat }) \pm 0.58(\text { sys }) \\
C_{F} & =1.35 \pm 0.07(\text { stat }) \pm 0.26(\text { sys }) .
\end{aligned}
$$

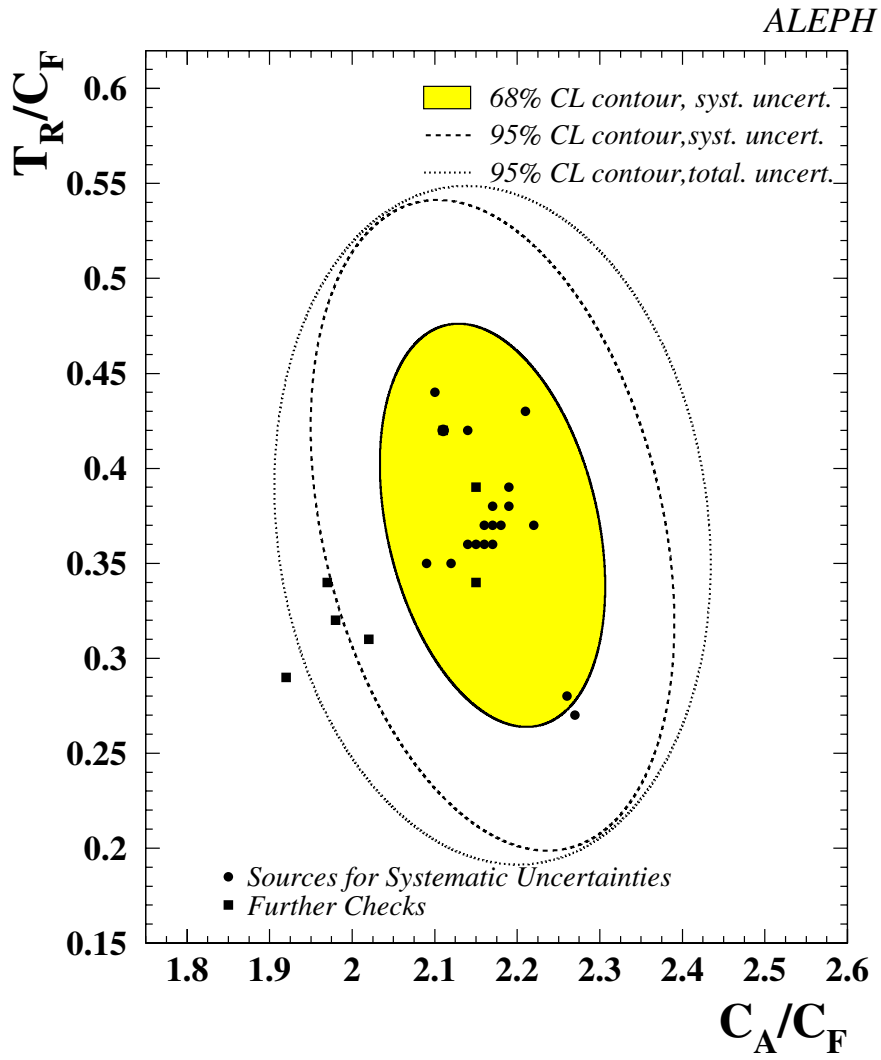

Fig. 9. $68 \%$ and $95 \%$ confidence level contours in the $(x=$ $\frac{C_{A}}{C_{F}}, y=\frac{T_{R}}{C_{F}}$ ) plane when taking into account systematic uncertainties only. The $95 \%$ confidence level contour with the total uncertainty is shown for comparison. Results for the various variations of the analysis are also indicated

Figure 8 shows that the measurement of the colour factor ratios is in agreement with the expectations from QCD $(x=2.25$ and $y=0.375)$. The agreement with previous measurements by ALEPH [22] and lately by OPAL $[3]$ is also observed.

Figure 9 shows the fitted colour factor ratios as found for the systematic variations considered in the analysis, as well as for most of the further checks listed in Sect. 6.4. It is observed that most of the variations are well covered by the $68 \%$ probability contour defined by the total systematic uncertainty.

\subsection{Light gluino hypothesis}

A study is carried out in order to test the hypothesis of the existence of a massless gluino [34]. The DEBRECEN program is used again in order to obtain the NLO perturbative prediction. This MC program provides not only the $B$ and $C$ functions for pure QCD, but also for QCD plus the contributions from a massless gluino (QCD+gluino hypothesis). Only the four-jet angular correlations are analyzed, since there is no consistent prediction for $R_{4}$, where gluino contributions are not available in the resummation terms. 
The measurement of the colour factors is repeated using as perturbative predictions for the four-jet angular correlations the ones given in (8). Two cases are considered. First, the $B$ and $C$ functions are taking into account only pure QCD configurations. Then the gluino contributions are also included in these functions, and the QCD beta function coefficients in (5) are changed to [35]

$$
\begin{aligned}
\beta_{0}= & \frac{11}{3} x-\frac{4}{3}\left(y N_{f}+x \frac{N_{\tilde{\mathrm{g}}}}{2}\right), \\
\beta_{1}= & \frac{17}{3} x^{2}-2\left(y N_{f}+x^{2} \frac{N_{\tilde{\mathrm{g}}}}{2}\right) \\
& -\frac{10}{3}\left(x y N_{f}+x^{2} \frac{N_{\tilde{\mathrm{g}}}}{2}\right),
\end{aligned}
$$

where $N_{\tilde{\mathrm{g}}}$ is the number of gluinos, set to 1 in this analysis. Hadronization and detector corrections are taken from the nominal analysis. At this stage, there is no MC program which models the gluino contributions to hadronization. All studies of systematic uncertainties described in Sect. 6.3 are repeated.

The results are

$$
\begin{gathered}
x=2.27 \pm 0.09 \text { (stat) } \pm 0.08(\text { sys }) \\
y=0.38 \pm 0.05(\text { stat }) \pm 0.07 \text { (sys) } \\
\left(\rho_{x y}\right)_{\text {total }}=-0.15
\end{gathered}
$$

for the pure QCD case, and

$$
\begin{gathered}
x=2.26 \pm 0.08(\text { stat }) \pm 0.07(\text { sys }) \\
y=0.15 \pm 0.06(\text { stat }) \pm 0.06 \text { (sys) } \\
\left(\rho_{x y}\right)_{\text {total }}=-0.19
\end{gathered}
$$

for the QCD+gluino hypothesis.

Figure 10 shows that these results exclude the existence of a massless gluino at more than $95 \%$ confidence level, since the measured colour factor ratios do not agree with the expectation of $\mathrm{SU}(3)$ anymore.

In a previous publication by ALEPH [22] a similar analysis allowed to set a limit on the light gluino mass. At that time only LO predictions existed for the fourjet angular correlations, both for massless and massive quarks. Thus a mass limit consistent at LO QCD could be extracted. The present study is based on NLO fourjet calculations, which are available only for the massless case. Therefore it is not possible to compute a consistent gluino mass limit in the context of NLO QCD, and the previously obtained limit is not superseded.

\section{Conclusions}

Two different kinds of measurements have been presented. First, three measurements of the strong coupling constant from the four-jet rate have been described. Second, the simultaneous measurement of the strong coupling constant and the QCD colour factors has been detailed. The analyses use ALEPH data from 1994 and 1995 and NLO predictions corrected to detector level.

The measurement of the strong coupling constant using NLO resummed predictions for the four-jet rate is the

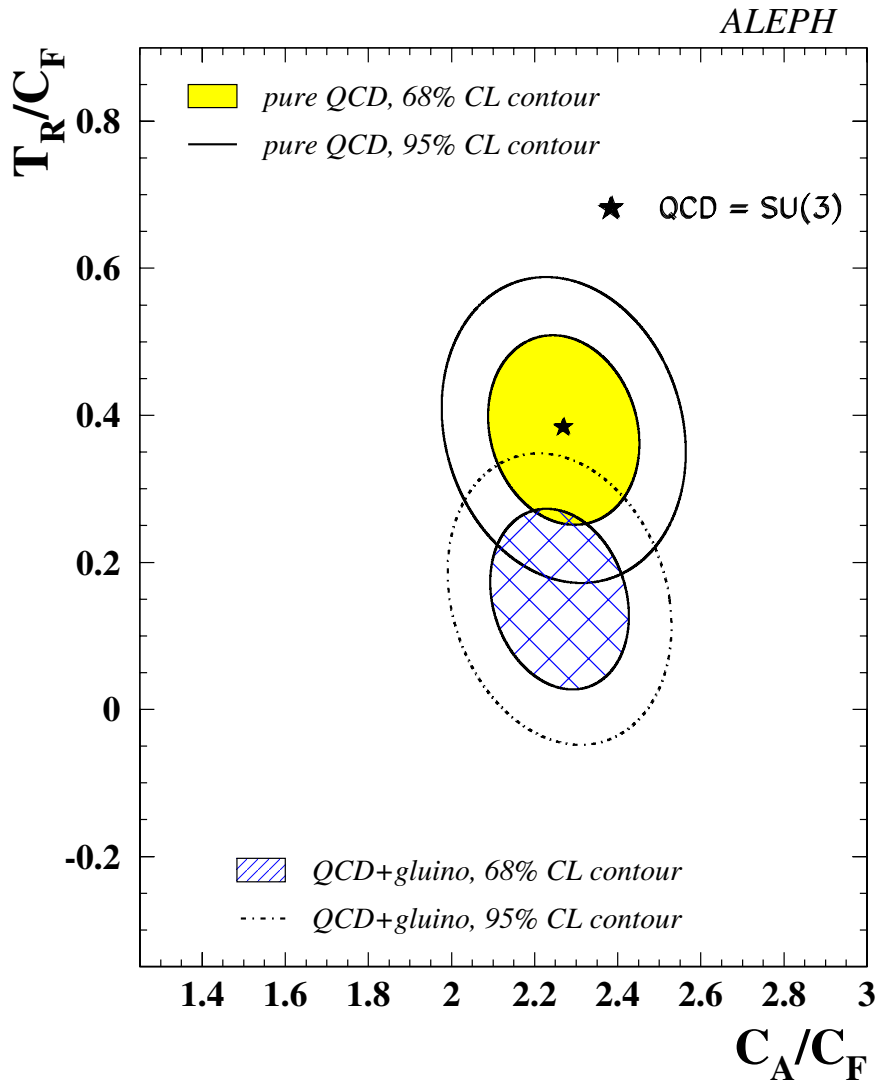

Fig. 10. $68 \%$ and $95 \%$ confidence level contours in the $(x=$ $\frac{C_{A}}{C_{F}}, y=\frac{T_{R}}{C_{F}}$ ) plane for the QCD and QCD+gluino hypotheses, based on four-jet angular correlations. The uncertainties include statistical as well as systematic errors

first measurement of $\alpha_{s}$ from a four-jet observable, yielding

$$
\alpha_{s}\left(M_{\mathrm{Z}}\right)=0.1170 \pm 0.0001 \text { (stat) } \pm 0.0013 \text { (sys) } .
$$

This result represents one of the most precise measurements of $\alpha_{s}$ at present. It is in good agreement with previous measurements from ALEPH and other collaborations which used two- and three-jet observables [26,1].

A stringent test of QCD was performed by measuring simultaneously the strong coupling constant and the colour factors. The test is based on NLO predictions, corrected to detector level, for five four-jet observables: the four-jet rate and four-jet angular correlations. New calculations and new Monte Carlo programs have allowed for a reduction in the total uncertainty. The results,

$$
\begin{aligned}
\alpha_{s}\left(M_{\mathrm{Z}}\right) & =0.119 \pm 0.006(\text { stat }) \pm 0.026(\text { sys }) \\
C_{A} & =2.93 \pm 0.14(\text { stat }) \pm 0.58(\text { sys }) \\
C_{F} & =1.35 \pm 0.07(\text { stat }) \pm 0.26(\text { sys })
\end{aligned}
$$

are in agreement with the expectation from QCD as well as with previous results from ALEPH [22]. A similar analysis, using the four-jet rate and the four-jet angular correlations, but also the differential two-jet rate, was performed by the OPAL Collaboration [3]. Again good agreement is found. 
The existence of a massless gluino has been excluded at more than $95 \%$ CL by repeating the measurement taking into account the gluino contributions in the NLO predictions. For this test the assumption is made that hadronization corrections are independent of the existence of the light gluino.

Acknowledgements. We wish to thank our colleagues in the CERN accelerator divisions for the successful operation of LEP. We are indebted to the engineers and technicians in all our institutions for their contribution to the excellent performance of ALEPH. Those of us from non-member countries thank CERN for its hospitality.

\section{References}

1. S. Bethke, Determination of the QCD Coupling $\alpha_{s}$, J. Phys. G 26, R27 (2000)

2. G. Dissertori, Measurements of the QCD Colour Factors at LEP, Nucl. Phys. B (Proc. Suppl) 65, 43 (1998)

3. OPAL Collaboration, A Simultaneous Measurement of the QCD Colour Factors and the Strong Coupling, Eur. Phys. J. C 20, 601 (2001)

4. A. Signer, L. Dixon, Electron-Positron Annihilation into Four Jets at Next-to-Leading Order in $\alpha_{s}$, Phys. Rev. Lett. 78, 811 (1997)

5. L. Dixon, A. Signer, Complete $\mathcal{O}\left(\alpha_{s}^{3}\right)$ Results for $e^{+} e^{-} \rightarrow$ $\gamma, Z \rightarrow$ Four Jets, Phys. Rev. D 56, 4031 (1997)

6. A. Signer, Next-to-leading order corrections to $e^{+} e^{-} \rightarrow$ four jets, hep-ph/9705218 (1997)

7. Z. Nagy, Z. Trócsányi, Next-to-Leading Oroder Calculation of Four-Jet Shape Variables, Phys. Rev. Lett. 79, 3604 (1997)

8. E.W.N. Glover, Jet Physics: Theoretical Overview, hepph/9805481 (1998)

9. Z. Nagy, Z. Trócsányi, Four-jet production in $e^{+} e^{-}$annihilation at next-to-leading order, Nucl. Phys. B (Proc. Suppl) 64, 63 (1998)

10. Z. Nagy, Z. Trócsányi, Four-jet angular distributions and colour charge measurements: leading order versus next-toleading order, Phys. Rev. D 57, 5793 (1998)

11. Z. Nagy, Z. Trócsányi, Next-to-leading order calculation of four-jet observables in electron-positron annihilation, Phys. Rev. D 59, 14020 (1999); Multijet rates in $e^{+} e^{-}$ annihilation: perturbation theory versus LEP data, Nucl. Phys. (Proc. Suppl.) 74, 44 (1999)

12. Z. Trócsányi, private communication (1999)

13. S. Catani, Yu.L. Dokshitzer, M. Olsson, G. Turnock, B.R. Webber, New clustering algorithm for multi-jet crosssections in $e^{+} e^{-}$annihilation, Phys. Lett. B 269, 432 (1991)

14. ALEPH Collaboration, Measurement of $\alpha_{s}$ from the structure of particle clusters produced in hadronic $\mathrm{Z}$ decays, Phys. Lett. B 257, 479 (1991)

15. N. Brown, W.J. Stirling, Finding jets and summing soft gluons: a new algorithm, Z. Phys. C 53, 629 (1992)
16. M. Bengtsson, P.M. Zerwas, Four-jet events in $e^{+} e^{-}$annihilation: testing the three-gluon vertex, Phys. Lett. B 208, 306 (1988); M. Bengtsson, Finding the 3-gluon vertex from 4-jet events in $e^{+} e^{-}$annihilation, Z. Phys. C 42, 75 (1989)

17. J.G. Körner, G. Schierholz, J. Willrodt, QCD predictions for four-jet final states in $e^{+} e^{-}$annihilation, Nucl. Phys. B 185, (1981) 365

18. O. Nachtmann, A. Reiter, A Test for the Gluon Selfcoupling in the Reactions $e^{+} e^{-} \rightarrow 4$ Jets and $Z^{0} \rightarrow$ 4Jets, Z. Phys. C 16, 45 (1982)

19. S. Bethke, A. Richter, P.M. Zerwas, Four-jet decays of the $Z^{0}$ : prospects of testing the triple gluon coupling, Phys. C 49, 59 (1991)

20. ALEPH Collaboration, ALEPH: a detector for electronpositron annihilations at LEP, Nucl. Instrum. Methods A 294, 121 (1990)

21. ALEPH Collaboration, Performance of the ALEPH detector at LEP, Nucl. Instrum. Methods A 360, 481 (1995)

22. ALEPH Collaboration, A measurement of the QCD colour factors and a limit on the light gluino, Z. Phys. C 76, 1 (1997)

23. T. Sjöstrand, Comp. Phys. Comm. 82, 74 (1994); T. Sjöstrand, P. Edén, C. Friberg, L. Lönnblad, G. Miu, S. Mrenna, E. Norrbin, Computer Phys. Commun. 135, 238 (2001)

24. C. Peterson et al., Scaling violations in inclusive $e^{+} e^{-}$annihilation spectra, Phys. Rev. D 27, 105 (1983)

25. "GEANT Detector Description and Simulation Tool", CERN Program Library, CERN-W5013 (1993)

26. ALEPH Collaboration, Studies of Quantum Chromodynamics with the ALEPH Detector, Phys. Rep. 294, 1 (1998)

27. G. Marchesini, B.R. Webber, G. Abbiendi, I.G. Knowles, M.H. Seymour, L. Stanco, Comp. Phys. Comm. 67, 465 (1992)

28. ALEPH Collaboration, Studies of QCD in $e^{+} e^{-} \rightarrow$ Hadrons at Ecm $=130$ and $136 \mathrm{GeV}$, Z. Phys. C 73, 409 (1997)

29. A. Ballestrero et al., Report of the QCD Working Group, hep-ph/0006259 (2000)

30. A. Ballestrero, private communication (2000)

31. G. Dissertori, A Simultaneous Measurement of the Strong Coupling Constant and the QCD Colour Factors, based on an Improved Theoretical Prediction for the Two-Jet Rate in $e^{+} e^{-}$Annihilations, Innsbruck University, ALEPH THESIS 97-009 (1997)

32. ALEPH Collaboration, A Measurement of the b-quark Mass from Hadronic Z Decays, Eur. Phys. J. C 18, 1 (2000)

33. J. Chýla, A. L. Kataev, Theoretical Ambiguities of QCD Predictions at the $Z^{0}$ Peak, hep-ex/9502383 (1995)

34. G. Farrar, Experiments to find or exclude a long-lived, light gluino, Phys. Rev. D 51, 3904 (1995)

35. L. Clavelli, P.W. Coulter, L.R. Surguladze, Gluino Contribution to the 3-loop QCD $\beta$ function in the Minimal Supersymmetric Standard Model, Phys. Lett. D 55, 4268 (1997) 